\title{
THE HOCHSCHILD COHOMOLOGY RING MODULO NILPOTENCE OF A MONOMIAL ALGEBRA
}

\author{
EDWARD L. GREEN, NICOLE SNASHALL, AND ØYVIND SOLBERG \\ Dedicated to Claus M. Ringel on the occasion of his sixtieth birthday
}

\begin{abstract}
For a finite dimensional monomial algebra $\Lambda$ over a field $K$ we show that the Hochschild cohomology ring of $\Lambda$ modulo the ideal generated by homogeneous nilpotent elements is a commutative finitely generated $K$ algebra of Krull dimension at most one. This was conjectured to be true for any finite dimensional algebra over a field in [13].
\end{abstract}

\section{INTRODUCTION}

Let $\Lambda$ be a finite dimensional algebra over a field $K$, with Jacobson radical $\mathfrak{r}$. Denote by $\Lambda^{e}$ the enveloping algebra $\Lambda^{\mathrm{op}} \otimes_{K} \Lambda$ of $\Lambda$. The Hochschild cohomology ring $\operatorname{HH}^{*}(\Lambda)$ of $\Lambda$ is given by $\operatorname{HH}^{*}(\Lambda)=\oplus_{i \geq 0} \operatorname{Ext}_{\Lambda^{e}}^{i}(\Lambda, \Lambda)$ with the Yoneda product. It is well-known that $\operatorname{HH}^{*}(\Lambda)$ is a graded commutative ring, so that when the characteristic of $K$ is different from two, then every homogeneous element of odd degree squares to zero. Hence $\mathrm{HH}^{*}(\Lambda) / \mathcal{N}$ is a commutative $K$-algebra, where $\mathcal{N}$ is the ideal generated by the homogeneous nilpotent elements. In [13] it was conjectured that $\mathrm{HH}^{*}(\Lambda) / \mathcal{N}$ is a finitely generated $K$-algebra. This paper is devoted to showing this for any finite dimensional monomial $K$-algebra $\Lambda$ and in addition that $\mathrm{HH}^{*}(\Lambda) / \mathcal{N}$ has Krull dimension at most one. Recall that $\Lambda$ is such a monomial algebra if $\Lambda=K \mathcal{Q} / I$ for some quiver $\mathcal{Q}$ and an ideal $I$ in $K \mathcal{Q}$ generated by monomials of length at least two.

This conjecture was earlier known to be true for Nakayama algebras with one relation ([13]), finite dimensional selfinjective indecomposable algebras of finite representation type over an algebraically closed field ([9]), any block of a group ring of a finite group $([4,7,14])$, and any block of a finite dimensional cocommutative Hopf algebra ([5] and see [13]). As a special case of our results we point out the following consequence of the work in [3]. Namely, the conjecture is true for a path algebra $K \mathcal{Q} / J^{2}$, where $J$ is the ideal generated by all the arrows.

Even though the proofs are technical, the strategy of the proof is easy to explain. The functor $\Lambda / \mathfrak{r} \otimes_{\Lambda}$ - from right $\Lambda^{e}$-modules to right $\Lambda$-modules induces a homomorphism of graded rings $\varphi_{\Lambda / \mathfrak{r}}: \operatorname{HH}^{*}(\Lambda) \rightarrow E(\Lambda)$, where $E(\Lambda)=\oplus_{i \geq 0} \operatorname{Ext}_{\Lambda}^{i}(\Lambda / \mathfrak{r}, \Lambda / \mathfrak{r})$. It is shown in [13] that $\operatorname{Im} \varphi_{\Lambda / \mathfrak{r}}$ is contained in the graded centre $Z_{\mathrm{gr}}(E(\Lambda))$ of $E(\Lambda)$, that is, the subring of $E(\Lambda)$ generated by all homogeneous elements $z$ such that $z g=(-1)^{|z||g|} g z$ for each homogeneous element $g$ in $E(\Lambda)$ where $|x|$ denotes the degree of a homogeneous element $x$. Furthermore the map $\varphi_{\Lambda / \mathfrak{r}}$ induces an inclusion $\overline{\varphi_{\Lambda / \mathrm{r}}}$ of $\mathrm{HH}^{*}(\Lambda) / \mathcal{N}$ into $Z_{\mathrm{gr}}(E(\Lambda)) / \mathcal{N}_{Z}$, where $\mathcal{N}_{Z}$ is the ideal in $Z_{\mathrm{gr}}(E(\Lambda))$ generated by homogeneous nilpotent elements. The method of proof is then as

Date: September 26, 2018. 
follows. First we show that $Z_{\mathrm{gr}}(E(\Lambda)) / \mathcal{N}_{Z}$ is a commutative finitely generated $K$ algebra, and secondly that beyond some degree the even parts of the graded rings $\mathrm{HH}^{*}(\Lambda) / \mathcal{N}$ and $Z_{\mathrm{gr}}(E(\Lambda)) / \mathcal{N}_{Z}$ are isomorphic via the map $\overline{\varphi_{\Lambda / \mathrm{r}}}$. It then follows easily that $\mathrm{HH}^{*}(\Lambda) / \mathcal{N}$ is a finitely generated $K$-algebra.

The precise relationship between $\operatorname{Im} \varphi_{\Lambda / \mathfrak{r}}$ and $E(\Lambda)$ for any finite dimensional $K$-algebra $\Lambda$ has been found by Bernhard Keller [12, Theorem 3.5]. The image $\operatorname{Im} \varphi_{\Lambda / \mathfrak{r}}$ is shown to be the $\mathbb{A}_{\infty}$-centre of $E(\Lambda)$. For further details see section 7 .

The paper is organized as follows. Let $\Lambda=K \mathcal{Q} / I$ be a monomial algebra. The first section is devoted to giving the multiplicative basis of $E(\Lambda)$ and the properties of this basis which are used throughout this paper. This is heavily based on the papers [1], [6] and [10]. The basis elements in $E(\Lambda)$ correspond to paths in $K \mathcal{Q}$. In section three it is shown that elements in $Z_{\mathrm{gr}}(E(\Lambda))$ are obtained from closed walks and equivalence classes of such. Non-nilpotent elements in $Z_{\mathrm{gr}}(E(\Lambda))$ of even degree are made up of closed walks which are a product of relations, and we call them tightly packed, extending walks. Definitions and properties of these closed walks are given in section two. The structure of non-nilpotent homogeneous elements in $Z_{\mathrm{gr}}(E(\Lambda))$ is analysed in further detail in the fourth section. The next section is devoted to characterizing the elements $z^{m}$ for homogeneous non-nilpotent elements $z$ in $Z_{\mathrm{gr}}(E(\Lambda))$ for an even positive integer $m$ less than or equal to the radical length of $\Lambda$. In the two last sections $Z_{\mathrm{gr}}(E(\Lambda))$ and $\mathrm{HH}^{*}(\Lambda)$ modulo the ideal generated by homogeneous nilpotent elements are shown to be finitely generated (commutative) $K$-algebras of Krull dimension at most one. The paper ends with a brief discussion of the relationship between $\operatorname{Im} \varphi_{\Lambda / \mathfrak{r}}$ and $E(\Lambda)$ for a general finite dimensional $K$-algebra and some examples illustrating our results.

\section{ACKNOWLEDGEMENT}

The authors acknowledge support from NSA and the Research Council of Norway, and support and hospitality from the departments of mathematics at the University of Leicester, Norwegian University of Science and Technology and Virginia Tech.

\section{Preliminaries}

In [10], it was shown that there is a multiplicative basis for the Ext algebra $E(\Lambda)$ for a finite dimensional monomial algebra $\Lambda=K \mathcal{Q} / I$. We now describe this basis in terms of the paths in $K \mathcal{Q}$. Arrows in a path are read from left to right. An arrow $a$ starts at the vertex $\mathfrak{o}(a)$ and ends at the vertex $\mathfrak{t}(a)$. We say that a path $p$ starts at the vertex $\mathfrak{o}(p)$ and ends at the vertex $\mathfrak{t}(p)$. We denote the length of a path $p$ by $\ell(p)$. A path $p$ is a prefix of a path $q$ if there is some path $p^{\prime}$ with $q=p p^{\prime}$. A path $p$ is a suffix of a path $q$ if there is some path $p^{\prime}$ with $q=p^{\prime} p$.

Fix some minimal generating set of monomials for the ideal $I$ and denote this set by $\mathcal{R}^{2}$.

We start by recalling some definitions and results from [1], [6] and [10] about overlaps of paths in $K \mathcal{Q}$. 
An overlap relation may be illustrated in the following way, where $P, Q, U, V$ are paths in $K \mathcal{Q}$ with $V Q=P U$.

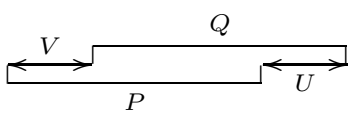

We now give the precise definition of an overlap relation.

Definition 1.1 ([6]). An overlap relation is a tuple $(P, Q, U, V)$ of paths in $K \mathcal{Q}$ such that

(i) $P U=V Q$, and

(ii) $1 \leq \ell(U)<\ell(Q)$ and $1 \leq \ell(V)<\ell(P)$.

In this case we say $P$ is overlapped by $Q$, or that $Q$ overlaps $P$.

Before giving the formal definition of a left overlap sequence and a right overlap sequence we again explain the concepts pictorially and by example.

A left overlap sequence is a sequence $\left(R_{2}, R_{3}, \ldots, R_{n}\right)$ with $R_{2}, R_{3}, \ldots, R_{n} \in \mathcal{R}^{2}$ that, in the case $n=5$, may be illustrated thus.

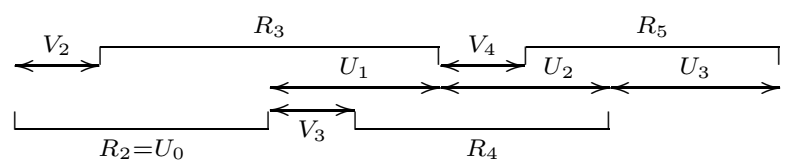

The underlying path is $U_{0} U_{1} U_{2} U_{3}$ and we have $U_{0} U_{1}=V_{2} R_{3}, U_{1} U_{2}=V_{3} R_{4}$ and $U_{2} U_{3}=V_{4} R_{5}$.

A right overlap sequence is a sequence $\left(R_{2}, R_{3}, \ldots, R_{n}\right)$ with $R_{2}, R_{3}, \ldots, R_{n} \in \mathcal{R}^{2}$ that, in the case $n=5$, may be shown in the following way.

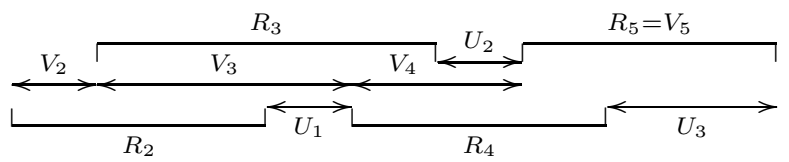

The underlying path is $V_{2} V_{3} V_{4} V_{5}$ and we have $V_{2} V_{3}=R_{2} U_{1}, V_{3} V_{4}=R_{3} U_{2}$ and $V_{4} V_{5}=R_{4} U_{3}$.

Example 1.2. Let $Q$ be the quiver given by

$$
1 \stackrel{a}{\longrightarrow} 2 \stackrel{b}{\longrightarrow} 3 \stackrel{c}{\longrightarrow} 4 \stackrel{d}{\longrightarrow} 5 \stackrel{e}{\longrightarrow} 6 \stackrel{f}{\longrightarrow} 7 \stackrel{g}{\longrightarrow} 8
$$

and relations $\mathcal{R}^{2}=\{a b c, b c d e, c d e f, e f g\}$. Then $(a b c, c d e f)$ is a left overlap sequence with path $a b c d e f$. The pair $(a b c, b c d e)$ is also a left overlap sequence and has path abcde.

We now give the formal definitions of these concepts.

Definition 1.3 ([6]). Let $n \geq 2$ and let $R_{2}, R_{3}, \ldots, R_{n} \in \mathcal{R}^{2}$.

(1) The sequence $\left(R_{2}, R_{3}, \ldots, R_{n}\right)$ is a left overlap sequence with data

$$
\left(U_{0}, \ldots, U_{n-2}, V_{2}, \ldots, V_{n-1}\right)
$$

if

(i) $U_{0}=R_{2}$, and

(ii) $\left(U_{i}, R_{i+3}, U_{i+1}, V_{i+2}\right)$ is an overlap relation for $0 \leq i \leq n-3$ (and in particular $\left.U_{i} U_{i+1}=V_{i+2} R_{i+3}\right)$. 
The path associated to a left overlap sequence $\left(R_{2}, R_{3}, \ldots, R_{n}\right)$ is the path $U_{0} \cdots U_{n-2}$.

(2) The sequence $\left(R_{2}, R_{3}, \ldots, R_{n}\right)$ is a right overlap sequence with data

$$
\left(U_{1}, \ldots, U_{n-2}, V_{2}, \ldots, V_{n}\right)
$$

if

(i) $V_{n}=R_{n}$, and

(ii) $\left(R_{i}, V_{i+1}, U_{i-1}, V_{i}\right)$ is an overlap relation for $2 \leq i \leq n-1$ (and in particular $\left.V_{i} V_{i+1}=R_{i} U_{i-1}\right)$.

The path associated to a right overlap sequence $\left(R_{2}, R_{3}, \ldots, R_{n}\right)$ is the path $V_{2} \cdots V_{n}$.

Remark. (1) Note that the paths $P$ and $Q$ in Definition 1.1 are not required to be in $\mathcal{R}^{2}$. However all the terms $R_{2}, R_{3}, \ldots, R_{n}$ in the definitions of a left overlap sequence and a right overlap sequence must lie in $\mathcal{R}^{2}$.

(2) If $R_{2}$ and $R_{3}$ are in $\mathcal{R}^{2}$ so that $R_{3}$ overlaps $R_{2}$, then $\left(R_{2}, R_{3}\right)$ is both a left overlap sequence and a right overlap sequence.

We now consider the algebra of Example 1.2 again to discuss maximal left overlap sequences and maximal right overlap sequences.

Example 1.4. Let $Q$ be the quiver given by

$$
1 \stackrel{a}{\longrightarrow} 2 \stackrel{b}{\longrightarrow} 3 \stackrel{c}{\longrightarrow} 4 \stackrel{d}{\longrightarrow} 5 \stackrel{e}{\longrightarrow} 6 \stackrel{f}{\longrightarrow} 7 \stackrel{g}{\longrightarrow} 8
$$

and relations $\mathcal{R}^{2}=\{a b c, b c d e, c d e f, e f g\}$. The left overlap sequence (abc, cdef) with path $a b c d e f$ is not a maximal left overlap sequence. However $(a b c, b c d e)$ with path $a b c d e$ is a maximal left overlap sequence. Also $(a b c, b c d e, e f g)$, illustrated as $a b c d e f g$, is a maximal left overlap sequence with path $a b c d e f g$. This path $a b c d e f g$ is also the path associated to the maximal right overlap sequence ( $a b c, c d e f, e f g$ ) or $a b c d e f g$.

The formal definitions are as follows.

Definition $1.5([6])$. Let $n \geq 2$ and let $R_{2}, R_{3}, \ldots, R_{n} \in \mathcal{R}^{2}$.

(1) The left overlap sequence $\left(R_{2}, R_{3}, \ldots, R_{n}\right)$ is a maximal left overlap sequence if

(i) $V_{2} R_{3} \neq P R Q$ for all nontrivial paths $P, Q$ and all $R \in \mathcal{R}^{2}$, and

(ii) for $i=3, \ldots, n-1, V_{i} R_{i+1} \neq P R Q$ for all nontrivial paths $Q$ and all $R \in \mathcal{R}^{2}$.

(2) The right overlap sequence $\left(R_{2}, R_{3}, \ldots, R_{n}\right)$ is a maximal right overlap sequence if

(i) $R_{n-1} U_{n-2} \neq P R Q$ for all nontrivial paths $P, Q$ and all $R \in \mathcal{R}^{2}$, and

(ii) for $i=1, \ldots, n-3, R_{i+1} U_{i} \neq P R Q$ for all nontrivial paths $P$ and all $R \in \mathcal{R}^{2}$.

In both cases we say the overlap sequence $\left(R_{2}, R_{3}, \ldots, R_{n}\right)$ is of length $n$.

For a monomial algebra, the paths associated to maximal left and maximal right overlap sequences are closely related as we recall from [1]. 
Theorem 1.6 ([1]). For a monomial algebra, the path associated to a maximal left overlap sequence $\left(R_{2}, R_{3}, \ldots, R_{n}\right)$ is the path associated to some maximal right overlap sequence $\left(\tilde{R}_{2}, \tilde{R}_{3}, \ldots, \tilde{R}_{n}\right)$, and vice versa.

Remark. It follows from the definitions that, in Theorem 1.6, we have $R_{2}=\tilde{R}_{2}$ and $R_{n}=\tilde{R}_{n}$. However $R_{i}$ need not equal $\tilde{R}_{i}$, for $i=3, \ldots, n-1$, as Example 1.4 illustrates.

The maximal left and right overlap sequences shall be omnipresent in all of our arguments, so that we introduce appropriate notation for these sets.

Definition 1.7. Define $\mathcal{R}^{n}$ to be the set of paths associated to the maximal left (or equivalently right) overlap sequences of length $n$ for $n \geq 2$. This is consistent with the definition already introduced of the set $\mathcal{R}^{2}$. Let $\mathcal{R}^{0}$ denote the set of vertices of $\mathcal{Q}$ and let $\mathcal{R}^{1}$ denote the set of arrows of $\mathcal{Q}$.

From now on we use lower case letters, $r, r_{i}$, to denote the elements of $\mathcal{R}^{2}$. By the term relation, we mean an element of the set $\mathcal{R}^{2}$, that is, of our fixed minimal set of monomial generators for the ideal $I$. Let $\left(r_{2}, r_{3}, \ldots, r_{n}\right)$ be a maximal left (or equivalently right) overlap sequence. We say that $r_{2}$ is the first relation and that $r_{n}$ is the last relation of the path corresponding to this overlap sequence.

The following are elementary properties of overlap sequences, and the proofs are straightforward and left to the reader.

Lemma 1.8. (1) The pair $\left(r_{2}, r_{3}\right)$ is a left overlap sequence if and only if $\left(r_{2}, r_{3}\right)$ is a right overlap sequence.

(2) If $\left(r_{2}, r_{3}\right)$ is a left overlap sequence, then there exists a maximal left overlap sequence $\left(r_{2}, \tilde{r}_{3}\right)$ such that the path associated to $\left(r_{2}, \tilde{r}_{3}\right)$ is a prefix of the path associated to $\left(r_{2}, r_{3}\right)$.

$\left(2^{\prime}\right)$ If $\left(r_{2}, r_{3}\right)$ is a right overlap sequence, then there exists a maximal right overlap sequence $\left(\tilde{r}_{2}, r_{3}\right)$ such that the path associated to $\left(\tilde{r}_{2}, r_{3}\right)$ is a suffix of the path associated to $\left(r_{2}, r_{3}\right)$.

(3) Suppose $S=\left(r_{2}, r_{3}, \ldots, r_{n}\right)$ is a maximal left overlap sequence and $S^{\prime}=\left(r_{2}, r_{3}, \ldots, r_{n}, r_{n+1}\right)$ is a left overlap sequence. Then there exists $\tilde{S}=\left(r_{2}, r_{3}, \ldots, r_{n}, \tilde{r}_{n+1}\right)$ which is a maximal left overlap sequence and the path associated to $\tilde{S}$ is a prefix of the path associated to $S^{\prime}$.

$\left(3^{\prime}\right)$ Suppose $S=\left(r_{3}, r_{4}, \ldots, r_{n}\right)$ is a maximal right overlap sequence and $S^{\prime}=\left(r_{2}, r_{3}, r_{4}, \ldots, r_{n}\right)$ is a right overlap sequence. Then there exists $\tilde{S}=\left(\tilde{r}_{2}, r_{3}, r_{4} \ldots, r_{n}\right)$ which is a maximal right overlap sequence and the path associated to $\widetilde{S}$ is a suffix of the path associated to $S^{\prime}$.

(4) If $S=\left(r_{2}, r_{3}, \ldots, r_{n}\right)$ is a left overlap sequence, then for all $i$ and $j$ with $1 \leq i<j \leq n-1$, we have that $S^{\prime}=\left(r_{i+1}, r_{i+2}, \ldots, r_{j+1}\right)$ is a left overlap sequence. Note that even if $S$ is maximal we need not have $S^{\prime}$ maximal. However $S^{\prime}$ is maximal in the case when $S$ is maximal and $i=1$.

$\left(4^{\prime}\right)$ If $S=\left(r_{2}, r_{3}, \ldots, r_{n}\right)$ is a right overlap sequence, then for all $i$ and $j$ with $1 \leq i<j \leq n-1$, we have that $S^{\prime}=\left(r_{i+1}, r_{i+2}, \ldots, r_{j+1}\right)$ is a right overlap sequence. Note that even if $S$ is maximal we need not have $S^{\prime}$ maximal. However $S^{\prime}$ is maximal in the case when $S$ is maximal and $j=n-1$.

The importance of the sets $\mathcal{R}^{n}$ comes from the fact that they describe a basis for the $n$-th graded part of the graded algebra $E(\Lambda)=\oplus_{i \geq 0} \operatorname{Ext}_{\Lambda}^{i}(\Lambda / \mathfrak{r}, \Lambda / \mathfrak{r})$. We 
explain this next. Following [6], fix a minimal $\Lambda$-projective resolution $\left(P^{*}, d^{*}\right)$ of the right $\Lambda$-module $\Lambda / \mathfrak{r}$ which is determined by $\bigcup_{m \geq 0} \mathcal{R}^{m}$. Then

$$
P^{m}=\coprod_{R_{j}^{m} \in \mathcal{R}^{m}} \mathfrak{t}\left(R_{j}^{m}\right) \Lambda .
$$

For each $R_{i}^{m} \in \mathcal{R}^{m}$ there is a corresponding element $g_{i}^{m}$ in $\operatorname{Ext}_{\Lambda}^{m}(\Lambda / \mathfrak{r}, \Lambda / \mathfrak{r})$. This element $g_{i}^{m}$ is represented by the map

$$
P^{m} \rightarrow \Lambda / \mathfrak{r}
$$

given by

$$
\mathfrak{t}\left(R_{j}^{m}\right) \mapsto \begin{cases}\mathfrak{t}\left(R_{i}^{m}\right) & \text { if } j=i, \\ 0 & \text { otherwise. }\end{cases}
$$

The set $\left\{g_{i}^{m}: R_{i}^{m} \in \mathcal{R}^{m}\right\}$ is a basis for $\operatorname{Ext}_{\Lambda}^{m}(\Lambda / \mathfrak{r}, \Lambda / \mathfrak{r})$ and is denoted by $\mathcal{G}^{m}$. From [10], the set $\bigcup_{m>0} \mathcal{G}^{m}$ forms a multiplicative basis for $\operatorname{Ext}_{\Lambda}^{*}(\Lambda / \mathfrak{r}, \Lambda / \mathfrak{r})$, that is, for $g_{i}^{m} \in \mathcal{G}^{m}$ and $g_{j}^{n} \in \mathcal{G}^{n}$, if $g_{i}^{m} g_{j}^{n} \neq 0$ then $g_{i}^{m} g_{j}^{n} \in \mathcal{G}^{m+n}$. For ease of notation and where no confusion will arise, we may denote an element of $\mathcal{G}^{m}$ by $g^{m}$ rather than $g_{i}^{m}$ for some $i$; in this case the corresponding path in $\mathcal{R}^{m}$ is denoted $R^{m}$. All relations between basis elements are generated by

(1) If $m=\sum_{i=1}^{s} m_{i}$ and if $R^{m_{1}} \cdots R^{m_{s}}$ is not in $\mathcal{R}^{m}$, then $g^{m_{1}} \cdots g^{m_{s}}=0$.

(2) If

$$
R^{m_{1}} \cdots R^{m_{s}}=R^{n_{1}} \cdots R^{n_{t}}
$$

is an element in $\mathcal{R}^{m}$ where $m=\sum_{i=1}^{s} m_{i}=\sum_{j=1}^{t} n_{j}$, then

$$
g^{m_{1}} \cdots g^{m_{s}}=g^{n_{1}} \cdots g^{n_{t}} .
$$

Note that if $m=\sum_{i=1}^{s} m_{i}$ and if $R^{m_{1}} \cdots R^{m_{s}} \in \mathcal{R}^{m}$, then $g^{m_{1}} \cdots g^{m_{s}} \in \mathcal{G}^{m}$ and so $g^{m_{1}} \cdots g^{m_{s}} \neq 0$.

In this paper we work extensively with the sets $\mathcal{R}^{n}$. Thus, for $R_{i}^{m}$ in $\mathcal{R}^{m}$ and $R_{j}^{n}$ in $\mathcal{R}^{n}$ with corresponding elements $g_{i}^{m}$ in $\mathcal{G}^{m}$ and $g_{j}^{n}$ in $\mathcal{G}^{n}$, we have $g_{i}^{m} g_{j}^{n} \neq 0$ if and only if $R_{i}^{m} R_{j}^{n}$ is in $\mathcal{R}^{m+n}$.

From the construction of this basis we have the following facts about the elements $g_{i}^{m}$ and the corresponding paths $R_{i}^{m}$. These results will be used throughout this paper.

Proposition 1.9 ([1, 6, 10]). For a finite dimensional monomial algebra $\Lambda=$ $K \mathcal{Q} / I$, the following properties hold.

(1) If $R_{i}^{u} R_{j}^{v}=R_{i}^{u} R_{k}^{w} \in \mathcal{R}^{u+v}$ then $v=w$ and $R_{j}^{v}=R_{k}^{w}$.

(2) If $R_{j}^{v} R_{i}^{u}=R_{k}^{w} R_{i}^{u} \in \mathcal{R}^{u+v}$ then $v=w$ and $R_{j}^{v}=R_{k}^{w}$.

(3) If $g_{i}^{u} g_{j}^{v}=g_{i}^{u} g_{k}^{w} \neq 0$ then $v=w$ and $g_{j}^{v}=g_{k}^{w}$.

(4) If $g_{j}^{v} g_{i}^{u}=g_{k}^{w} g_{i}^{u} \neq 0$ then $v=w$ and $g_{j}^{v}=g_{k}^{w}$.

Proposition 1.10 ([10]). For a finite dimensional monomial algebra $\Lambda=K \mathcal{Q} / I$, if $g_{1}^{m_{1}} \ldots g_{s}^{m_{s}}=g_{1^{\prime}}^{m_{1}} \ldots g_{s^{\prime}}^{m_{s}} \neq 0$ then $g_{i}^{m_{i}}=g_{i^{\prime}}^{m_{i}}$ for $i=1, \ldots, s$.

The next two results give further properties of the basis for $E(\Lambda)$, which we use later.

Proposition 1.11. For a finite dimensional monomial algebra $\Lambda=K \mathcal{Q} / I$, the following properties hold.

(1) If $R_{i}^{m} p=R_{j}^{m} q$ for some paths $p$ and $q$, then $R_{i}^{m}=R_{j}^{m}$ and $p=q$. 
(2) If $p R_{i}^{m}=q R_{j}^{m}$ for some paths $p$ and $q$, then $R_{i}^{m}=R_{j}^{m}$ and $p=q$.

Proof. (1) Suppose $R_{i}^{m} p=R_{j}^{m} q$ but that $R_{i}^{m} \neq R_{j}^{m}$. Let $R_{i}^{m}$ have maximal left overlap sequence $\left(r_{2}, r_{3}, \ldots, r_{m}\right)$ and let $R_{j}^{m}$ have maximal left overlap sequence $\left(\tilde{r}_{2}, \tilde{r}_{3}, \ldots, \tilde{r}_{m}\right)$. Then by construction, $r_{2}=\tilde{r}_{2}$, and there is some $k$ such that $r_{2}=\tilde{r}_{2}, \ldots, r_{k}=\tilde{r}_{k}$ but $r_{k+1} \neq \tilde{r}_{k+1}$. Without loss of generality we may assume that $\tilde{r}_{k+1}$ starts after $r_{k+1}$. Thus we have the following diagram.

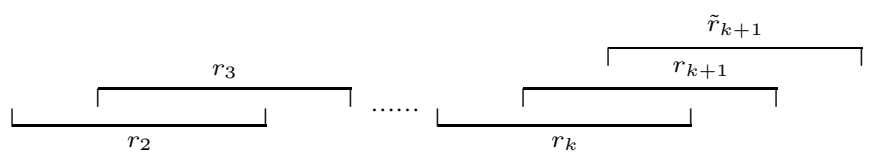

But this contradicts $\left(\tilde{r}_{2}, \tilde{r}_{3}, \ldots, \tilde{r}_{m}\right)$ being a maximal left overlap sequence. Hence $R_{i}^{m}=R_{j}^{m}$ and thus $p=q$.

(2) The proof is similar.

Proposition 1.12. For a finite dimensional monomial algebra $\Lambda=K \mathcal{Q} / I$, if $g^{m} g^{n} \neq 0$ and $n \geq 2$, then $g^{m} g^{2} \neq 0$ where $g^{2}$ is the basis element corresponding to the first relation of $R^{n}$.

Proof. Let $\left(r_{2}, r_{3}, \ldots, r_{m}, r_{m+1}, \ldots, r_{m+n}\right)$ be a maximal left overlap sequence for $R^{m} R^{n}$. Then from Lemma 1.8 , we have that $\left(r_{2}, r_{3}, \ldots, r_{m}\right)$ is a maximal left overlap sequence for $R^{m}$. Since $\mathfrak{t}\left(R^{m}\right)=\mathfrak{o}\left(R^{n}\right)$, the relation $r_{m+2}$ is the first relation of $R^{n}$. Thus $\left(r_{2}, r_{3}, \ldots, r_{m}, r_{m+1}, r_{m+2}\right)$ is a maximal left overlap sequence for $R^{m} r_{m+2}$. Hence $R^{m} r_{m+2} \in \mathcal{R}^{m+2}$ and so $g^{m} g^{2} \neq 0$ where $g^{2}$ is the basis element corresponding to the first relation of $R^{n}$.

In multiplying the basis elements for $E(\Lambda)$ the following concept is central.

Definition $1.13([6])$. Let $\Lambda=K \mathcal{Q} / I$ be a finite dimensional monomial algebra.

(1) For each $R_{i}^{m} \in \mathcal{R}^{m}, m \geq 1$, there exists a unique path $t$ and unique element $R_{k}^{m-1} \in \mathcal{R}^{m-1}$ with $R_{i}^{m}=R_{k}^{m-1} t$. We call $t$ the tail path of $R_{i}^{m}$ and write $t=t\left(R_{i}^{m}\right)$.

(2) For each $R_{i}^{m} \in \mathcal{R}^{m}, m \geq 1$, there exists a unique path $b$ and unique element $R_{j}^{m-1} \in \mathcal{R}^{m-1}$ with $R_{i}^{m}=b R_{j}^{m-1}$. We call $b$ the beginning path of $R_{i}^{m}$ and write $b=b\left(R_{i}^{m}\right)$.

We end this section with some remarks about closed walks in a quiver.

Definition 1.14. A closed walk in $\mathcal{Q}$ is a non-trivial path $C$ in $K \mathcal{Q}$ such that $C=e C e$ for some vertex $e$.

We do not make any assumptions with this terminology as to whether or not $C$ is a nonzero element in the algebra $\Lambda$.

Lemma 1.15. Let $p, q$ and $r$ be walks in a quiver $\mathcal{Q}$, and let $a_{1}, \ldots, a_{t}$ be arrows in $\mathcal{Q}$. Suppose that $p q=q r$. If $p=a_{1} a_{2} \cdots a_{t}$, then $q=p^{s} a_{1} \cdots a_{i}$ and $r=$ $a_{i+1} \cdots a_{t} a_{1} \cdots a_{i}$ for some $0 \leq i<t$ and $s \geq 0$, where the case $i=0$ implies that $q=p^{s}$ and $r=p$. Moreover $p$ and $r$ are closed walks in $\mathcal{Q}$. 


\section{Tightly PACKED MAXIMAL OVERLAP SEQUENCES}

Our strategy of proof is to first analyse the graded centre of $E(\Lambda)$ modulo nilpotence. Recall that the graded centre $Z_{\mathrm{gr}}(E(\Lambda))$ of $E(\Lambda)$ is the subring generated by all homogeneous elements $z$ in $E(\Lambda)$ such that $z g=(-1)^{|z||g|} g z$ for each homogeneous element $g$ in $E(\Lambda)$. The elements in $E(\Lambda)$ are described by the basis $\cup_{m \geq 0} \mathcal{G}^{m}$. The path $R^{m}$ corresponding to a basis vector $g^{m}$ need not be a product of relations; however it turns out that all the elements in $Z_{\mathrm{gr}}(E(\Lambda))$ are associated to paths which are products of relations. Such paths we call tightly packed. In this section we define and give some properties of tightly packed paths which will be needed for our characterization of the non-nilpotent elements of $Z_{\mathrm{gr}}(E(\Lambda))$.

Definition 2.1. A path $p$ in $K \mathcal{Q}$ is tightly packed if $p=r_{1} r_{2} \cdots r_{n}$ for some $r_{i} \in \mathcal{R}^{2}$, $i=1, \ldots, n$. We say that $r_{1}, r_{2}, \ldots, r_{n}$ are the relations in $p$.

The next four results give basic properties of tightly packed paths.

Proposition 2.2. Suppose $R^{2 n}$ in $\mathcal{R}^{2 n}$ is tightly packed with $R^{2 n}=r_{2} r_{4} \cdots r_{2 m}$ for some $m$ and $r_{2}, r_{4}, \ldots, r_{2 m} \in \mathcal{R}^{2}$. Then $m=n$ and the maximal left (resp. right) overlap sequence for $R^{2 n}$ is of the form $\left(r_{2}, r_{3}, r_{4}, \ldots, r_{2 n}\right)$.

Proof. Let $\left(\tilde{r}_{2}, \tilde{r}_{3}, \ldots, \tilde{r}_{2 n}\right)$ be the maximal left overlap sequence for $R^{2 n}$. Then the path corresponding to this maximal left overlap sequence is of the form $\tilde{r}_{2} p$ and hence $\tilde{r}_{2}=r_{2}$. Now $\mathfrak{t}\left(r_{2}\right)=\mathfrak{o}\left(r_{4}\right)$ so, by maximality of the left overlap sequence, we have $\tilde{r}_{4}=r_{4}$. By induction $\tilde{r}_{6}=r_{6}, \ldots, \tilde{r}_{2 n}=r_{2 n}$. Then the path associated to the maximal left overlap sequence is $r_{2} r_{4} \cdots r_{2 n}$ and so $m=n$.

Proposition 2.3. Suppose the path $p=r_{2} r_{4} \cdots r_{2 n}$ is tightly packed and that there is some left overlap sequence $\left(r_{2}, r_{3}, r_{4}, \ldots, r_{2 n}\right)$. Then $p \in \mathcal{R}^{2 n}$.

Proof. We show that there is some maximal left overlap sequence $\left(r_{2}, \tilde{r}_{3}, r_{4}, \ldots, \tilde{r}_{2 n-1}, r_{2 n}\right)$ with path $p$. Since $\left(r_{2}, r_{3}, r_{4}, \ldots, r_{2 n}\right)$ is a left overlap sequence, the relation $r_{3}$ overlaps $r_{2}$. Thus there is some maximal left overlap $\tilde{r}_{3}$ with $r_{2}$, and so $\tilde{r}_{3}$ ends at or before $r_{3}$ ends. Now $\mathfrak{t}\left(r_{2}\right)=\mathfrak{o}\left(r_{4}\right)$ so $\left(r_{2}, \tilde{r}_{3}, r_{4}\right)$ is a maximal left overlap sequence. The relation $r_{5}$ overlaps $r_{4}$ and starts at or after the end of $\tilde{r}_{3}$ as the diagram illustrates.

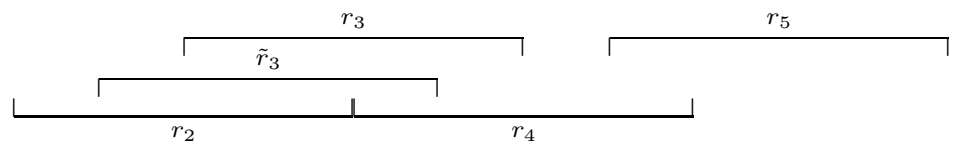

Thus there is some maximal overlap $\tilde{r}_{5}$ with $r_{4}$ such that $\tilde{r}_{5}$ ends at or before $r_{5}$ ends and $\left(r_{2}, \tilde{r}_{3}, r_{4}, \tilde{r}_{5}\right)$ is a maximal left overlap sequence. Continuing in this way we have a maximal left overlap sequence $\left(r_{2}, \tilde{r}_{3}, r_{4}, \tilde{r}_{5}, \ldots, \tilde{r}_{2 n-1}, r_{2 n}\right)$ with path $p$. Hence $p$ is in $\mathcal{R}^{2 n}$.

Corollary 2.4. Suppose $R^{2 n}=r_{2} r_{4} \cdots r_{2 n}$ is tightly packed and $R^{2 n} \in \mathcal{R}^{2 n}$. Then for each $1 \leq i \leq j \leq n$, the subpath $r_{2 i} r_{2 i+2} \cdots r_{2 j}$ is in $\mathcal{R}^{2(j-i)+2}$.

Proof. From Proposition 2.3, there is a maximal left overlap sequence $\left(r_{2}, r_{3}, r_{4}, \ldots r_{2 n}\right)$ for $R^{2 n}$. From Lemma $1.8,\left(r_{2 i}, r_{2 i+1}, \ldots, r_{2 j}\right)$ is a left overlap sequence for $r_{2 i} r_{2 i+2} \cdots r_{2 j}$. The result now follows by applying Proposition 2.3 again. 
Proposition 2.5. Suppose $R^{2 n}$ is in $\mathcal{R}^{2 n}$ and $\left(R^{2 n}\right)^{u}$ is in $\mathcal{R}^{2 n u}$ for some $u \geq 1$. Suppose also that $\left(R^{2 n}\right)^{u}$ is tightly packed. Then $R^{2 n}$ is tightly packed.

Proof. Suppose $\left(R^{2 n}\right)^{u}$ in $\mathcal{R}^{2 n u}$ is tightly packed. From Proposition 2.2, we may write $\left(R^{2 n}\right)^{u}=r_{2} r_{4} \cdots r_{2 n u}$ with $r_{2}, r_{4}, \ldots r_{2 n u} \in \mathcal{R}^{2}$. Then Corollary 2.4 gives that $r_{2} r_{4} \cdots r_{2 n}$ is in $\mathcal{R}^{2 n}$. Now, as paths, $\left(r_{2} r_{4} \cdots r_{2 n}\right)\left(r_{2 n+2} r_{2 n+4} \cdots r_{2 n u}\right)=\left(R^{2 n}\right)^{u}=$ $R^{2 n}\left(\left(R^{2 n}\right)^{(u-1)}\right)$. So from Proposition 1.11 we have $r_{2} r_{4} \cdots r_{2 n}=R^{2 n}$. Hence $R^{2 n}$ is tightly packed.

Remark. If $\left(R^{2 n}\right)^{u} \in \mathcal{R}^{2 n u}$ for some $u \geq 2$, then $\left(R^{2 n}\right)^{u}$ is the path corresponding to some element of $\mathcal{R}^{2 n u}$, and in particular, $R^{2 n}$ is a closed walk.

When multiplying elements in $E(\Lambda)$, the tail and the beginning of a path corresponding to a basis element are important for deciding the product of two basis elements. In particular we are interested in non-nilpotent elements so that the tails of powers of basis elements need to be considered. The next three results discuss the tails of powers of tightly packed paths, culminating by showing that the tails of high enough powers of tightly packed paths become equal if the path is "non-nilpotent".

Proposition 2.6. Suppose $R^{2 n}$ in $\mathcal{R}^{2 n}$ is tightly packed and $R^{2 n} R^{2 n}$ is in $\mathcal{R}^{4 n}$. Then $t\left(R^{2 n} R^{2 n}\right)$ is a suffix of $t\left(R^{2 n}\right)$.

Proof. Let $R^{2 n}$ have maximal left overlap sequence $\left(r_{2}, r_{3}, \ldots, r_{2 n}\right)$, and let $\left(\tilde{r}_{2}, \tilde{r}_{3}, \ldots, \tilde{r}_{4 n}\right)$ be the maximal left overlap sequence for $R^{2 n} R^{2 n}$. Since $R^{2 n}$ is tightly packed, $R^{2 n}=r_{2} r_{4} \cdots r_{2 n}$. It follows that $R^{2 n} R^{2 n}$ is tightly packed and so

$$
r_{2} r_{4} \cdots r_{2 n} r_{2} r_{4} \cdots r_{2 n}=R^{2 n} R^{2 n}=\tilde{r}_{2} \tilde{r}_{4} \cdots \tilde{r}_{4 n} .
$$

Thus $\tilde{r}_{i}=\tilde{r}_{i+2 n}=r_{i}$ for $i=2,4,6, \ldots, 2 n$. Moreover, $\left(\tilde{r}_{2}, \ldots, \tilde{r}_{2 n}\right)$ is a maximal left overlap sequence for $R^{2 n}$ and so $\tilde{r}_{2}=r_{2}, \tilde{r}_{3}=r_{3}, \ldots, \tilde{r}_{2 n}=r_{2 n}$.

We now consider $\tilde{r}_{2 n+1}$. Viewing paths from left to right we have the following maximal left overlap sequence
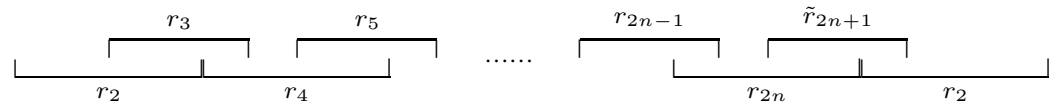

where either $\tilde{r}_{2 n+1}$ does not end after $r_{3}$ begins or $\tilde{r}_{2 n+1}$ ends strictly after $r_{3}$ begins. In the first case, if $\tilde{r}_{2 n+1}$ does not end after $r_{3}$ begins, then $\tilde{r}_{2 n+1}$ is situated as follows:

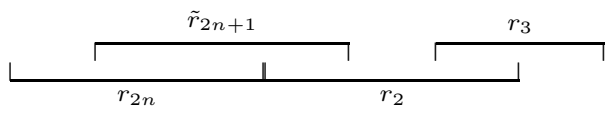

By maximality of the left overlap sequence $\left(r_{2}, r_{3}, \ldots, r_{2 n}\right)$ for $R^{2 n}$, the relation $r_{3}$ is the first relation that overlaps $r_{2}$. Hence $\tilde{r}_{2 n+3}=r_{3}$. Then the maximal left overlap sequence for $R^{2 n} R^{2 n}$ continues as the sequence for $R^{2 n}$, that is, $\tilde{r}_{2 n+i}=\tilde{r}_{i}$ for $i=1,2, \ldots, 2 n$. Thus $t\left(R^{2 n} R^{2 n}\right)=t\left(R^{2 n}\right)$.

So now consider the second case, that is, suppose $\tilde{r}_{2 n+1}$ ends strictly after $r_{3}$ begins so that $\tilde{r}_{2 n+1}$ is now situated as follows

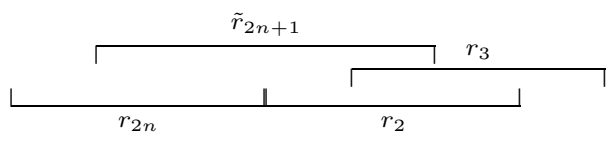


Now $R^{2 n} R^{2 n}$ is in $\mathcal{R}^{4 n}$ so there exists some relation $\tilde{r}_{2 n+3}$ along the path and this relation must overlap $r_{2}$, ending strictly after $r_{3}$ ends, and thus we have

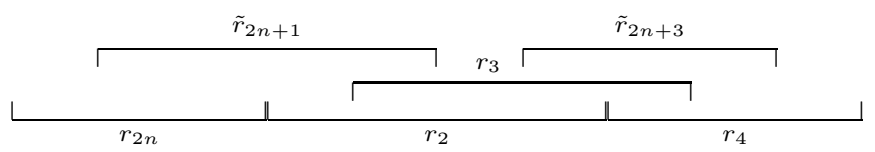

Now either $\tilde{r}_{2 n+3}$ does not end after $r_{5}$ begins, in which case $t\left(R^{2 n} R^{2 n}\right)=t\left(R^{2 n}\right)$ as above, or $\tilde{r}_{2 n+3}$ ends strictly after $r_{5}$ begins, in which case we consider $\tilde{r}_{2 n+5}$.

Continue inductively until we reach $\tilde{r}_{4 n-3}$. Either $\tilde{r}_{4 n-3}$ does not end after $r_{2 n-1}$ begins, in which case $t\left(R^{2 n} R^{2 n}\right)=t\left(R^{2 n}\right)$ as above, or $\tilde{r}_{4 n-3}$ ends strictly after $r_{2 n-1}$ begins, in which case $\tilde{r}_{4 n-3}$ is situated as follows:

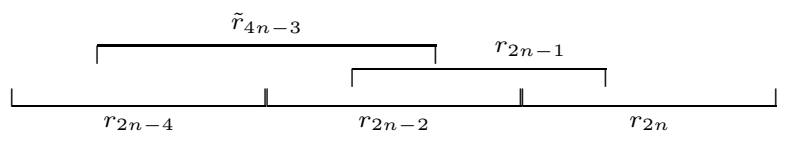

Then $\tilde{r}_{4 n-1}$ must end strictly after $r_{2 n-1}$ ends. Thus we have

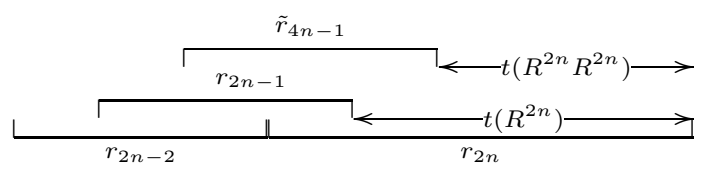

and so $t\left(R^{2 n} R^{2 n}\right)$ is a proper suffix of $t\left(R^{2 n}\right)$.

Remark. The corresponding proof for the beginning paths shows that $b\left(R^{2 n} R^{2 n}\right)$ is a prefix of $b\left(R^{2 n}\right)$.

Proposition 2.7. Suppose $R^{2 n}$ in $\mathcal{R}^{2 n}$ is tightly packed and $\left(R^{2 n}\right)^{(k+1)}$ is in $\mathcal{R}^{2 n(k+1)}$ for some $k \geq 1$. Then $t\left(\left(R^{2 n}\right)^{(k+1)}\right)$ is a suffix of $t\left(\left(R^{2 n}\right)^{k}\right)$.

Proof. Note that if $\left(R^{2 n}\right)^{(k+1)}$ is in $\mathcal{R}^{2 n(k+1)}$, for some $k \geq 1$, then $\left(R^{2 n}\right)^{k}$ is in $\mathcal{R}^{2 n k}$ by Corollary 2.4. The proof is now by induction with the case $k=1$ being Proposition 2.6. The rest of the proof is similar and is left to the reader.

Definition 2.8. A closed walk $R^{2 n}$ is extending if $\left(R^{2 n}\right)^{s} \in \mathcal{R}^{2 n s}$ for all $s \geq 1$.

Proposition 2.9. Suppose $R^{2 n}$ is an extending closed walk. Then there exists $u \geq 1$ such that $t\left(\left(R^{2 n}\right)^{u}\right)=t\left(\left(R^{2 n}\right)^{(u+v)}\right)$ for all $v \geq 1$. Moreover $u \leq \operatorname{rl}(\Lambda)-1$ where $\operatorname{rl}(\Lambda)$ is the radical length of $\Lambda$.

Proof. From Proposition 2.6 the lengths of the tail paths $t\left(\left(R^{2 n}\right)^{k}\right)$ for $k \geq 1$ form a decreasing sequence, that is,

$$
\ell\left(t\left(R^{2 n}\right)\right) \geq \ell\left(t\left(\left(R^{2 n}\right)^{2}\right)\right) \geq \ell\left(t\left(\left(R^{2 n}\right)^{3}\right)\right) \geq \cdots .
$$

Since each successive tail path is a suffix of the previous tail path, there exists some $u$ with $t\left(\left(R^{2 n}\right)^{u}\right)=t\left(\left(R^{2 n}\right)^{(u+1)}\right)$. Moreover each tail path has length at least 1 , so $u \leq \operatorname{rl}(\Lambda)-1$.

Let $\left(r_{2}, r_{3}, \ldots, r_{2 n u}\right)$ be a maximal left overlap sequence for $\left(R^{2 n}\right)^{u}$, so that we have the following diagram:
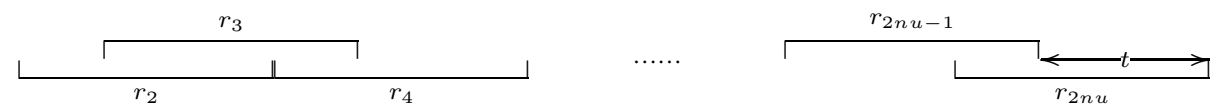
Then there is a maximal left overlap sequence $\left(r_{2}, r_{3}, \ldots, r_{2 n u}, \tilde{r}_{2 n u+1}, \ldots, \tilde{r}_{2 n(u+1)}\right)$ for $\left(R^{2 n}\right)^{(u+1)}$ with $\tilde{r}_{2 n u+2}=r_{2}, \tilde{r}_{2 n u+4}=r_{4}, \ldots, \tilde{r}_{2 n(u+1)}=r_{2 n}$ since $R^{2 n}$ is tightly packed.

The relation $\tilde{r}_{2 n u+1}$ overlaps $r_{2 n u}$ and starts after $r_{2 n u-1}$ ends; we consider successively the overlaps $\tilde{r}_{2 n u+1}, \tilde{r}_{2 n u+3}, \ldots, \tilde{r}_{2 n(u+1)-3}$, as in the proof of Proposition 2.6. Since $t\left(\left(R^{2 n}\right)^{(u+1)}\right)=t\left(\left(R^{2 n}\right)^{u}\right)$, the relation $\tilde{r}_{2 n(u+1)-1}$ must end precisely where $r_{2 n u-1}$ ends, giving a maximal left overlap sequence

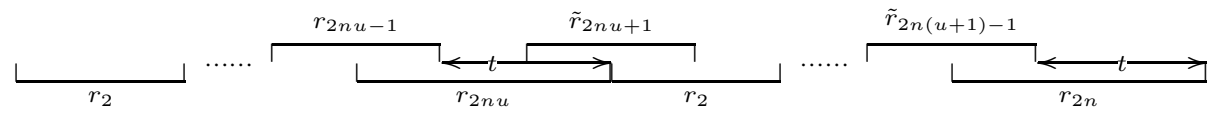

Note that $\tilde{r}_{2 n(u+1)-1}$ is necessarily equal to $r_{2 n u-1}$. Then, repeating the overlaps $\tilde{r}_{2 n u+1}, \ldots, \tilde{r}_{2 n(u+1)-1}$, shows that

$$
\left(r_{2}, r_{3}, \ldots, r_{2 n u}, \tilde{r}_{2 n u+1}, \ldots, \tilde{r}_{2 n(u+1)-1}, \tilde{r}_{2 n u+1}, \ldots, \tilde{r}_{2 n(u+1)-1}\right)
$$

is a maximal left overlap sequence for $\left(R^{2 n}\right)^{(u+2)}$. Thus $t\left(\left(R^{2 n}\right)^{(u+2)}\right)=$ $t\left(\left(R^{2 n}\right)^{(u+1)}\right)$. Hence, by induction, $t\left(\left(R^{2 n}\right)^{u}\right)=t\left(\left(R^{2 n}\right)^{(u+v)}\right)$ for all $v \geq 1$.

As we have seen above, the tail paths of the powers of an extending closed walk always become equal after the power $\operatorname{rl}(\Lambda)-1$ of the walk. However we could have equality for an earlier exponent. This gives rise to the next definition, which is of the concept of being left or right stable.

Definition 2.10. Let $R^{2 n} \in \mathcal{R}^{2 n}$ be an extending closed walk.

(1) The path $R^{2 n}$ is left $j$-stable if

(a) $j$ is odd, and

(b) whenever $s$ is such that $2 n s \geq j+1+2 n$, then $r_{j}=r_{2 n+j}$ and $r_{j+1}=$ $r_{2 n+j+1}$ where $\left(r_{2}, r_{3}, \ldots, r_{2 n s}\right)$ is the maximal left overlap sequence for $\left(R^{2 n}\right)^{s}$.

(2) The path $R^{2 n}$ is right $j$-stable if

(a) $j$ is odd, and

(b) whenever $s$ is such that $2 n s \geq j+1+2 n$, then $\tilde{r}_{2 n s+2-j}=\tilde{r}_{2 n s+2-j-2 n}$ and $\tilde{r}_{2 n s+1-j}=\tilde{r}_{2 n s+1-j-2 n}$ where $\left(\tilde{r}_{2}, \tilde{r}_{3}, \ldots, \tilde{r}_{2 n s}\right)$ is the maximal right overlap sequence for $\left(R^{2 n}\right)^{s}$.

The rest of this section is devoted to describing the tail path and the beginning path of a power of a tightly packed, extending closed walk.

Proposition 2.11. (1) If $R^{2 n}$ is a tightly packed, left $j$-stable, closed walk with $j \leq 2 n(s-1)-1$ and with maximal left overlap sequence $\left(r_{2}, r_{3}, \ldots, r_{2 n s}\right)$ for $\left(R^{2 n}\right)^{s}$, then $r_{k}=r_{2 n+k}$ for all $j \leq k \leq 2 n(s-1)$.

(2) If $R^{2 n}$ is a tightly packed, right $j$-stable, closed walk with maximal right overlap sequence $\left(\tilde{r}_{2}, \tilde{r}_{3}, \ldots, \tilde{r}_{2 n s}\right)$ for $\left(R^{2 n}\right)^{s}$, then $\tilde{r}_{k}=\tilde{r}_{k-2 n}$ for all $2 n+$ $2 \leq k \leq j$.

Proof. (1) Since $R^{2 n}$ is tightly packed, $r_{2 i}=r_{2 n+2 i}$ for $i=1, \ldots, n(s-1)$. Let $j=2 i+1$ where $R^{2 n}$ is left $j$-stable. Then $r_{2 n+2 i+1}=r_{2 i+1}, r_{2 n+2 i+2}=$ $r_{2 i+2}$ and $r_{2 n+2 i+4}=r_{2 i+4}$. By maximality of the left overlap sequence $\left(r_{2}, r_{3}, \ldots, r_{2 n s}\right)$, it follows that $r_{2 i+3}=r_{2 n+2 i+3}$. Continuing inductively, $r_{2 i+5}=$ $r_{2 n+2 i+5}, \ldots, r_{2 n(s-1)-1}=r_{2 n s-1}$. Hence $r_{k}=r_{2 n+k}$ for all $j \leq k \leq 2 n(s-1)$.

(2) The proof is similar. 
Proposition 2.12. Let $R^{2 n} \in \mathcal{R}^{2 n}$ be a tightly packed, extending closed walk. Then the following statements hold.

(1) The path $R^{2 n}$ is left $j$-stable for some $j \geq 3$, and $t\left(\left(R^{2 n}\right)^{v}\right)=t\left(\left(R^{2 n}\right)^{(v+1)}\right)$ for each $v$ such that $j<2 n v$.

(2) The path $R^{2 n}$ is right $j$-stable for some $j \geq 3$, and $b\left(\left(R^{2 n}\right)^{v}\right)=b\left(\left(R^{2 n}\right)^{(v+1)}\right)$ for each $v$ such that $j<2 n v$.

Proof. (1) By Proposition 2.9 there is some $u \geq 1$ such that $t\left(\left(R^{2 n}\right)^{u}\right)=$ $t\left(\left(R^{2 n}\right)^{(u+1)}\right)$, and so $r_{2 n u-1}=r_{2 n(u+1)-1}$ where $\left(r_{2}, r_{3}, \ldots, r_{2 n(u+1)}\right)$ is the maximal left overlap sequence for $\left(R^{2 n}\right)^{(u+1)}$. Since $R^{2 n}$ is tightly packed, $r_{2 n}=r_{2 n u}=$ $r_{2 n(u+1)}$. Thus $R^{2 n}$ is left $(2 n u-1)$-stable.

Now suppose that $R^{2 n}$ is left $j$-stable, and let $v$ be such that $j<2 n v$. Let $\left(r_{2}, r_{3}, \ldots, r_{2 n(v+1)}\right)$ be the maximal left overlap sequence for $\left(R^{2 n}\right)^{(v+1)}$. Then by Proposition 2.11, we have $r_{k}=r_{2 n+k}$ for all $k$, with $j \leq k \leq 2 n v$. In particular, $r_{2 n v-1}=r_{2 n(v+1)-1}$. Since $R^{2 n}$ is tightly packed, $r_{2 n v}=r_{2 n(v+1)}$, and so $t\left(\left(R^{2 n}\right)^{v}\right)=t\left(\left(R^{2 n}\right)^{(v+1)}\right)$.

The proof of (2) is similar and is left to the reader.

Definition 2.13. Let $R^{2 n}$ be an extending closed walk. We say $R^{2 n}$ stabilizes at $u \geq 1$ if $R^{2 n}$ is left and right $j$-stable for some $j \leq 2 n u-1$.

Proposition 2.14. Let $R^{2 n}$ be a tightly packed extending closed walk which stabilizes at $u \geq 1$. Then, for all $k \geq 1$ and $0 \leq i<2 n$,

(1) if $\left(R^{2 n}\right)^{(u+k)}=R^{2 n(u+k-1)+i} q$ and $\left(R^{2 n}\right)^{2 u}=R^{2 n u+i} q^{\prime}$ where $R^{2 n(u+k-1)+i} \in$ $\mathcal{R}^{2 n(u+k-1)+i}$ and $R^{2 n+i} \in \mathcal{R}^{2 n+i}$ then

$$
t\left(R^{2 n(u+k-1)+i}\right)=t\left(R^{2 n u+i}\right),
$$

and

(2) if $\left(R^{2 n}\right)^{(u+k)}=q R^{2 n(u+k-1)+i}$ and $\left(R^{2 n}\right)^{2 u}=q^{\prime} R^{2 n u+i}$ where $R^{2 n(u+k-1)+i} \in$ $\mathcal{R}^{2 n(u+k-1)+i}$ and $R^{2 n+i} \in \mathcal{R}^{2 n+i}$ then

$$
b\left(R^{2 n(u+k-1)+i}\right)=b\left(R^{2 n u+i}\right) .
$$

Proof. By Proposition 2.12, $r_{2 n u-1}=r_{2 n(u+k)-1}$ and $r_{2 n u}=r_{2 n(u+k)}$ for all $k \geq 1$, where $\left(r_{2}, r_{3}, \ldots, r_{2 n(u+k)}\right)$ is the maximal left overlap sequence for $\left(R^{2 n}\right)^{(u+k)}$. From Proposition 2.11, we have $r_{2 n u+i}=r_{2 n u+2 n k+i}$ for all $k \geq 1$ and $0 \leq i<2 n$. But then, with the above notation, and since $\left(r_{2}, r_{3}, \ldots, r_{2 n(u+k-1)+i}\right)$ is a maximal left overlap sequence for $R^{2 n(u+k-1)+i}$ and $\left(r_{2}, r_{3}, \ldots, r_{2 n u+i}\right)$ is a maximal left overlap sequence for $R^{2 n u+i}$, it follows that $t\left(R^{2 n(u+k-1)+i}\right)=t\left(R^{2 n u+i}\right)$ for all $k \geq 1$ and $0 \leq i<2 n$.

The proof of (2) for beginning paths is similar.

\section{The EquiValence Relation}

In this section we begin our study of the graded centre of the Ext algebra modulo the ideal generated by the homogeneous nilpotent elements. The graded centre of the Ext algebra is denoted $Z_{\mathrm{gr}}(E(\Lambda))$ and is the subring of $E(\Lambda)$ generated by all homogeneous elements $z$ such that $z g=(-1)^{|z||g|} g z$ for each homogeneous element $g$ in $E(\Lambda)$.

Let $Z^{m}(E(\Lambda))$ denote the vector space of homogeneous elements of degree $m$ in the graded centre $Z_{\mathrm{gr}}(E(\Lambda))$. Thus $Z_{\mathrm{gr}}(E(\Lambda))=\oplus_{m \geq 0} Z^{m}(E(\Lambda))$. For ease of 
notation we do not repeat the subscript gr on the homogeneous spaces $Z^{m}(E(\Lambda))$; note that we do not use the notation $Z$ to represent any ungraded centre of a ring.

We consider here elements in $Z_{\mathrm{gr}}(E(\Lambda))$ of even degree, noting that if the characteristic of the field is not 2, then every element of $Z_{\mathrm{gr}}(E(\Lambda))$ is of even degree. We return to this point with a discussion of elements of odd degree in $Z_{\mathrm{gr}}(E(\Lambda))$ at the end of section 4 .

Let $z$ be a homogeneous element of degree $2 n$ in $Z_{\mathrm{gr}}(E(\Lambda))$, that is, let $z$ be in $Z^{2 n}(E(\Lambda))$. Then, using the multiplicative basis for $E(\Lambda)$, we may write $z=$ $\sum_{i=1}^{l} \alpha_{i} g_{i}^{2 n}$ for some $\alpha_{i} \in K \backslash\{0\}$. For each $i=1, \ldots, l$, we say that $g_{i}^{2 n}$ occurs in $z$.

The element $z$ is in $Z_{\mathrm{gr}}(E(\Lambda))$ and so for each basis element $g_{j}^{0}$ of $\mathcal{G}^{0}$, we have $g_{j}^{0} z=z g_{j}^{0}$. Thus, for each $i=1, \ldots, l$, there is some vertex $e_{v_{i}}$ with $R_{i}^{2 n}=$ $e_{v_{i}} R_{i}^{2 n} e_{v_{i}}$. Hence we may write $z=\sum_{i=1}^{l} \alpha_{i} g_{i}^{2 n}$, where the $R_{i}^{2 n}$ are closed walks in the quiver, and $\alpha_{i} \in K \backslash\{0\}$.

The following result and the ideas in its proof are used throughout this paper.

Lemma 3.1. If $i \neq j$ then $g_{i}^{2 n} g_{j}^{2 n}=0$ whenever $g_{i}^{2 n}$ and $g_{j}^{2 n}$ occur in $z$.

Proof. Suppose that $g_{i}^{2 n} g_{j}^{2 n} \neq 0$. We show first that $z g_{j}^{2 n} \neq 0$. For suppose for contradiction that $z g_{j}^{2 n}=0$. Then, since $z=\sum_{k=1}^{l} \alpha_{k} g_{k}^{2 n}$ with $\alpha_{k} \in K \backslash\{0\}$, we have $\sum_{k=1}^{l} \alpha_{k} g_{k}^{2 n} g_{j}^{2 n}=0$. Thus $\sum_{g_{k}^{2 n} g_{j}^{2 n} \neq 0} \alpha_{k} g_{k}^{2 n} g_{j}^{2 n}=0$, and this is not the empty sum since $g_{i}^{2 n} g_{j}^{2 n} \neq 0$. Now each of the terms $g_{k}^{2 n} g_{j}^{2 n}$ where $g_{k}^{2 n} g_{j}^{2 n} \neq 0$, is a basis element in $E(\Lambda)$ since we have a multiplicative basis. Thus $\left\{g_{k}^{2 n} g_{j}^{2 n}: g_{k}^{2 n} g_{j}^{2 n} \neq 0\right\}$ is a linearly independent set by Proposition 1.9. Hence each coefficient $\alpha_{k}$ in the $\operatorname{sum} \sum_{g_{k}^{2 n} g_{j}^{2 n} \neq 0} \alpha_{k} g_{k}^{2 n} g_{j}^{2 n}$ must be zero, and in particular $\alpha_{i}=0$. But this is a contradiction. Thus $z g_{j}^{2 n} \neq 0$.

Now $z \in Z_{\mathrm{gr}}(E(\Lambda))$, so $z g_{j}^{2 n}=g_{j}^{2 n} z$. Thus

$$
\sum_{k=1}^{l} \alpha_{k} g_{k}^{2 n} g_{j}^{2 n}=\sum_{k=1}^{l} \alpha_{k} g_{j}^{2 n} g_{k}^{2 n}
$$

and so

$$
\sum_{g_{k}^{2 n} g_{j}^{2 n} \neq 0} \alpha_{k} g_{k}^{2 n} g_{j}^{2 n}=\sum_{g_{j}^{2 n} g_{k}^{2 n} \neq 0} \alpha_{k} g_{j}^{2 n} g_{k}^{2 n} .
$$

Now each $g_{k}^{2 n} g_{j}^{2 n}$ and $g_{j}^{2 n} g_{k}^{2 n}$ in this last equality is a basis element in $E(\Lambda)$ so since $g_{i}^{2 n} g_{j}^{2 n} \neq 0$, there is some unique $i^{\prime}$ with $g_{i}^{2 n} g_{j}^{2 n}=g_{j}^{2 n} g_{i^{\prime}}^{2 n}$ and $\alpha_{i}=\alpha_{i^{\prime}}$. Hence from Proposition 1.10 we have $R_{i}^{2 n}=R_{j}^{2 n}$ and so $i=j$.

Thus if $i \neq j$ then $g_{i}^{2 n} g_{j}^{2 n}=0$.

Now we introduce an equivalence relation on certain closed walks in $\mathcal{Q}$.

Definition 3.2. (1) Let $p$ be any path and let $q$ be a closed walk in a quiver $\mathcal{Q}$. Then $p$ lies on $q$ if $p$ is a subpath of $q^{s}$ for some $s \geq 1$.

(2) Let $p$ and $q$ be closed walks in a quiver $\mathcal{Q}$. We define a relation by $p \sim q$ if $p$ lies on $q$ and $q$ lies on $p$.

Remark. (1) It is easy to verify that this is an equivalence relation.

(2) If $q$ and $q^{\prime}$ are both in the same equivalence class, then a path $p$ lies on $q$ if and only if $p$ lies on $q^{\prime}$. 
(3) Throughout this paper, any reference to an equivalence relation is referring to that of Definition 3.2.

The next result uses the above definition to show that the problem of describing the elements in $Z_{\mathrm{gr}}(E(\Lambda))$ can be reduced to each equivalence class of closed walks.

Proposition 3.3. Let $z \in Z^{2 n}(E(\Lambda))$. Then $z=\sum_{i=1}^{r} z_{i}$ with $z_{i}=\sum_{g_{j}^{2 n} \in \mathcal{F}_{i}} \alpha_{j} g_{j}^{2 n}$, where $\alpha_{j} \in K \backslash\{0\}$, each $\mathcal{F}_{i}$ is a subset of $\mathcal{E}_{i}$, and the sets $\mathcal{E}_{i}$ are distinct equivalence classes of closed walks in $K \mathcal{Q}$. Moreover each $z_{i}$ is in $Z_{\mathrm{gr}}(E(\Lambda))$.

Proof. Let $z \in Z^{2 n}(E(\Lambda))$. We have seen that we may write $z=\sum_{i=1}^{l} \alpha_{i} g_{i}^{2 n}$, where the $R_{i}^{2 n}$ are closed walks in the quiver and $\alpha_{i} \in K \backslash\{0\}$. Using the equivalence relation in Definition 3.2, each $R_{i}^{2 n}$ lies in some equivalence class. Thus we may write $z=\sum_{i=1}^{r} z_{i}$ with $z_{i}=\sum_{g_{j}^{2 n} \in \mathcal{F}_{i}} \alpha_{j} g_{j}^{2 n}$, where each $\mathcal{F}_{i}$ is a subset of $\mathcal{E}_{i}$, and the sets $\mathcal{E}_{i}$ are distinct equivalence classes of closed walks.

Now we show that each $z_{i}$ is itself in $Z_{\text {gr }}(E(\Lambda))$. Let $g^{m}$ be an arbitrary basis element of $E(\Lambda)$ of degree $m$ such that $g^{m} z_{i} \neq 0$. Thus there exists $j$ so that $g_{j}^{2 n}$ occurs in $z_{i}$ and $g^{m} g_{j}^{2 n} \neq 0$. Using the arguments in the proof of Lemma 3.1, we have that $g^{m} z \neq 0$, and so there exists $j^{\prime}$ such that $g_{j^{\prime}}^{2 n}$ occurs in $z, g^{m} g_{j}^{2 n}=g_{j^{\prime}}^{2 n} g^{m}$ and $\alpha_{j}=\alpha_{j^{\prime}}$. Then, by Lemma $1.15, R_{j}^{2 n}$ and $R_{j^{\prime}}^{2 n}$ are in the same equivalence class $\mathcal{E}_{i}$, and thus $g_{j^{\prime}}^{2 n}$ occurs in $z_{i}$. Continuing in this way and using the corresponding argument with $z_{i} g^{m} \neq 0$ shows that nonzero terms in $g^{m} z_{i}$ and $z_{i} g^{m}$ pair up with the same coefficients. Thus $g^{m} z_{i}=z_{i} g^{m}$. Hence $z_{i} \in Z_{\mathrm{gr}}(E(\Lambda))$ for all $i$.

Remark. (1) Note that we do not assume that all $g_{j}^{2 n}$, for which $R_{j}^{2 n}$ lies in the equivalence class $\mathcal{E}_{i}$, occur in $z$ with nonzero coefficient.

(2) It follows from Lemma 3.1 that $z_{i} z_{j}=0$ for $i \neq j$ and thus $z^{v}=\sum_{i=1}^{r} z_{i}^{v}$ for any $v \geq 1$.

From Proposition 3.3, we may suppose without loss of generality that if $z \in$ $Z^{2 n}(E(\Lambda))$ then $z=\sum_{g_{j}^{2 n} \in \mathcal{F}} \alpha_{j} g_{j}^{2 n}$ where $\mathcal{F}$ is a subset of the equivalence class $\mathcal{E}$. We say that such an element $z$ is associated to the equivalence class $\mathcal{E}$.

The next stage is to determine more precisely the subset $\mathcal{F}$ of the equivalence class $\mathcal{E}$.

\section{Non-Nilpotent ElEments of the GRADED CENTRE}

Let $\mathcal{N}_{Z}$ denote the ideal of $Z_{\mathrm{gr}}(E(\Lambda))$ generated by the homogeneous nilpotent elements. Then $\mathcal{N}_{Z}$ is a graded ideal and we write $\mathcal{N}_{Z}=\oplus_{m \geq 0} \mathcal{N}_{Z}^{m}$, with $\mathcal{N}_{Z}^{m}$ being the vector space of nilpotent homogeneous elements of degree $m$ in $Z_{\mathrm{gr}}(E(\Lambda))$. Now the quotient $Z_{\mathrm{gr}}(E(\Lambda)) / \mathcal{N}_{Z}$ has the decomposition

$$
Z_{\text {gr }}(E(\Lambda)) / \mathcal{N}_{Z}=\oplus_{m \geq 0}\left(Z^{m}(E(\Lambda)) / \mathcal{N}_{Z}^{m}\right) .
$$

If $\operatorname{char} K \neq 2$, then all elements of odd degree in $Z_{\mathrm{gr}}(E(\Lambda))$ are nilpotent. Thus, in every characteristic, $Z_{\mathrm{gr}}(E(\Lambda)) / \mathcal{N}_{Z}$ is a commutative $K$-algebra.

Fix some equivalence class $\mathcal{E}$. Again we consider elements in $Z_{\text {gr }}(E(\Lambda))$ of even degree. Using Proposition 3.3, it is enough to consider elements $z \in Z^{2 n}(E(\Lambda))$ which are non-nilpotent and are associated to the equivalence class $\mathcal{E}$. Write $z=$ $\sum_{i=1}^{l} \alpha_{i} g_{i}^{2 n}$ with $\alpha_{i} \in K \backslash\{0\}$ and $R_{i}^{2 n} \in \mathcal{E}$. 
Now partition the set $\{1, \ldots, l\}$ into two subsets $\mathcal{I}$ and $\mathcal{I}^{\prime}$ where $\mathcal{I}=\left\{i:\left(g_{i}^{2 n}\right)^{k} \neq\right.$ 0 for all $k \geq 1\}$ and $\mathcal{I}^{\prime}=\left\{i:\left(g_{i}^{2 n}\right)^{k}=0\right.$ for some $\left.k \geq 1\right\}$. Then we may write $z=\sum_{i \in \mathcal{I}} \alpha_{i} g_{i}^{2 n}+y$ where $y=\sum_{i \in \mathcal{I}^{\prime}} \alpha_{i} g_{i}^{2 n}$. Using Lemma 3.1, $y$ is nilpotent.

Now, from Proposition 2.9, we may choose $u$ large enough so that $y^{u}=0$ and, for each $i \in \mathcal{I}$, we have $t\left(\left(R_{i}^{2 n}\right)^{u}\right)=t\left(\left(R_{i}^{2 n}\right)^{(u+1)}\right)$ and $b\left(\left(R_{i}^{2 n}\right)^{u}\right)=b\left(\left(R_{i}^{2 n}\right)^{(u+1)}\right)$. Then $z^{u}=\sum_{i \in \mathcal{I}} \alpha_{i}^{u} g_{i}^{2 n u}$ (from Lemma 3.1). Note that $z^{u}$ is also in $Z_{\mathrm{gr}}(E(\Lambda))$. We keep this notation for the rest of this section.

The following example shows an element of the graded centre for which we require $u=2$.

Example 4.1. Let $\Lambda=K \mathcal{Q} / I$ where $\mathcal{Q}$ is the quiver

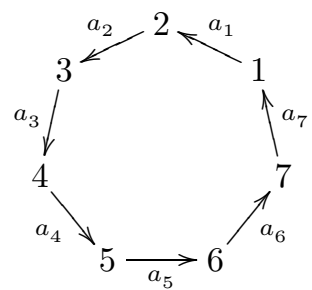

and

$$
I=\left\langle a_{1} a_{2} a_{3} a_{4}, a_{2} a_{3} a_{4} a_{5}, a_{4} a_{5} a_{6}, a_{5} a_{6} a_{7}, a_{6} a_{7} a_{1} a_{2}, a_{7} a_{1} a_{2} a_{3}\right\rangle
$$

The elements of the set $\mathcal{R}^{4}$ are

$$
\begin{array}{lll}
R_{1}^{4}=a_{1} a_{2} a_{3} a_{4} a_{5} a_{6} a_{7} & \text { with } & t\left(R_{1}^{4}\right)=a_{6} a_{7} \\
R_{2}^{4}=a_{4} a_{5} a_{6} a_{7} a_{1} a_{2} a_{3} & \text { with } & t\left(R_{2}^{4}\right)=a_{1} a_{2} a_{3} \\
R_{3}^{4}=a_{5} a_{6} a_{7} a_{1} a_{2} a_{3} a_{4} & \text { with } & t\left(R_{3}^{4}\right)=a_{3} a_{4} \\
R_{4}^{4}=a_{7} a_{1} a_{2} a_{3} a_{4} a_{5} a_{6} & \text { with } & t\left(R_{4}^{4}\right)=a_{5} a_{6} \\
R_{5}^{4}=a_{2} a_{3} a_{4} a_{5} a_{6} a_{7} a_{1} a_{2} & \text { with } & t\left(R_{5}^{4}\right)=a_{7} a_{1} a_{2} .
\end{array}
$$

It may be verified that $R_{1}^{4}, R_{2}^{4}, R_{3}^{4}$ and $R_{4}^{4}$ are all tightly packed extending closed walks. Note that $R_{5}^{4}$ is not a closed path so the element $g_{5}^{4}$ is nilpotent. Let $z=g_{1}^{4}+g_{2}^{4}+g_{3}^{4}+g_{4}^{4}$. Then $z \in Z_{\text {gr }}(E(\Lambda))$ and is non-nilpotent.

Now

$$
\mathcal{R}^{8}=\left\{\left(R_{1}^{4}\right)^{2},\left(R_{2}^{4}\right)^{2},\left(R_{3}^{4}\right)^{2},\left(R_{4}^{4}\right)^{2}\right\}
$$

with

$$
t\left(\left(R_{1}^{4}\right)^{2}\right)=a_{7}, t\left(\left(R_{2}^{4}\right)^{2}\right)=a_{1} a_{2} a_{3}, t\left(\left(R_{3}^{4}\right)^{2}\right)=a_{4}, t\left(\left(R_{1}^{4}\right)^{2}\right)=a_{5} a_{6} .
$$

Thus if $u=1$, then $t\left(\left(R_{1}^{4}\right)^{u}\right) \neq t\left(\left(R_{1}^{4}\right)^{(u+1)}\right)$. However, for $s \geq 3$, if $\left(r_{2}, r_{3}, \ldots, r_{4 s}\right)$ is a maximal left overlap sequence for $\left(R_{1}^{4}\right)^{s}$ then $r_{7}=r_{11}=a_{4} a_{5} a_{6}$ and $r_{8}=r_{12}=$ $a_{5} a_{6} a_{7}$ and so $R_{1}^{4}$ is left 7 -stable. Moreover $R_{1}^{4}$ is also right 7 -stable. Indeed, each of $R_{1}^{4}, R_{2}^{4}, R_{3}^{4}$ and $R_{4}^{4}$ is both left 7 -stable and right 7 -stable. So, for $u=2$, we have $t\left(\left(R_{i}^{4}\right)^{u}\right)=t\left(\left(R_{i}^{4}\right)^{(u+1)}\right)$ for $i=1, \ldots, 4$.

Note that in this example $Z_{\mathrm{gr}}(E(\Lambda)) / \mathcal{N}_{Z} \cong K[\bar{z}]$.

Remark. Suppose that $g_{i}^{2 n}$ is in $\mathcal{G}^{2 n}$ and that $\left(g_{i}^{2 n}\right)^{u} \neq 0$ for some $u \geq 1$. Then $\left(g_{i}^{2 n}\right)^{u}$ is in $\mathcal{G}^{2 n u}$ and so $\left(R_{i}^{2 n}\right)^{u}$ is the path corresponding to some element of $\mathcal{R}^{2 n u}$. We write $R_{i}^{2 n u}$ for the element of $\mathcal{R}^{2 n u}$ with path $\left(R_{i}^{2 n}\right)^{u}$, and will use this notation throughout the rest of the paper.

The next results describe the paths $R_{i}^{2 n u}$ for $i \in \mathcal{I}$.

Proposition 4.2. Suppose $g_{i}^{2 n u}$ occurs in $z^{u}$. Then the following statements hold. 
(1) The path $R_{i}^{2 n u}$ is tightly packed and may be expressed in the form

$$
R_{i}^{2 n u}=r_{i, 2} r_{i, 4} \cdots r_{i, 2 n u}
$$

where $r_{i, 2 l}$ is the path corresponding to some basis element $g_{i, 2 l}^{2} \in \mathcal{G}^{2}$. Thus the $r_{i, 2 l}$ are relations lying on every representative of $\mathcal{E}$.

(2) $R_{i}^{2 n}$ is tightly packed with

$$
R_{i}^{2 n}=r_{i, 2} r_{i, 4} \cdots r_{i, 2 n}
$$

(3) The relations in (1) are such that $r_{i, 2 c n+2 d}=r_{i, 2 d}$ for $c=1, \ldots, u-1, d=$ $1, \ldots, n-1$.

Proof. (1) Without loss of generality suppose $i=1$. Let $r_{1,2}$ be the first relation in $R_{1}^{2 n u}$ and let $g_{1,2}^{2}$ be the basis element in $E(\Lambda)$ with path $r_{1,2}$. Then $\left(g_{1}^{2 n u}\right)^{2} \neq 0$ and so Proposition 1.12 gives $g_{1}^{2 n u} g_{1,2}^{2} \neq 0$. Thus, using the ideas in the proof of Lemma 3.1, $z^{u} g_{1,2}^{2} \neq 0$ and there is some $i \in \mathcal{I}$ with $g_{1}^{2 n u} g_{1,2}^{2}=g_{1,2}^{2} g_{i}^{2 n u}$. Hence $R_{1}^{2 n u} r_{1,2}=r_{1,2} R_{i}^{2 n u}$. Now the first relation of $R_{i}^{2 n u}$ is $r_{i, 2}$, and thus $r_{1,2} R_{i}^{2 n u}$ is a path with prefix $r_{1,2} r_{i, 2}$. Hence $R_{1}^{2 n u}$ has prefix $r_{1,2} r_{i, 2}$. Let $r_{1,4}=r_{i, 2}$ so that $R_{1}^{2 n u}$ has prefix $r_{1,2} r_{1,4}$.

Continuing inductively shows that $R_{1}^{2 n u}=r_{1,2} \cdots r_{1,2 n u} p$ for some path $p$, and that there is a maximal left overlap sequence with path $r_{1,2} r_{1,4} \cdots r_{1,2 n u}$. By Proposition 2.3, $r_{1,2} r_{1,4} \cdots r_{1,2 n u} \in \mathcal{R}^{2 n u}$ and so, by Proposition 1.11, the path $p$ is a vertex. Thus $R_{1}^{2 n u}=r_{1,2} \cdots r_{1,2 n u}$ and so $R_{1}^{2 n u}$ is a product of relations. Hence, for each $g_{i}^{2 n u}$ occurring in $z^{u}, R_{i}^{2 n u}$ is a product of relations and we write $R_{i}^{2 n u}=r_{i, 2} r_{i, 4} \cdots r_{i, 2 n u}$. Thus $R_{i}^{2 n u}$ is tightly packed.

(2) and (3) now follow from Proposition 2.5.

Lemma 4.3. With the notation of Proposition 4.2, let $r_{i, 2}$ be a relation in $R_{i}^{2 n}$, for some $i \in \mathcal{I}$ and $h=1, \ldots, n$. Then $r_{i, 2 h}$ is the first relation of some $R_{j}^{2 n}$ with $j \in \mathcal{I}$. Moreover $\alpha_{i}=\alpha_{j}$.

Proof. Since $i \in \mathcal{I}$, the element $g_{i}^{2 n u}$ occurs in $z^{u}$ and $g_{i}^{2 n u} g_{i}^{2 n} \neq 0$. Thus from Proposition 1.12, $g_{i}^{2 n u} g_{i, 2}^{2} \neq 0$ where $g_{i, 2}^{2}$ is the basis element corresponding to the relation $r_{i, 2}$. Using the arguments of Lemma 3.1, we have that $z^{u} g_{i, 2}^{2} \neq 0$ and that there is some $j \in \mathcal{I}$ with $g_{i}^{2 n u} g_{i, 2}^{2}=g_{i, 2}^{2} g_{j}^{2 n u}$ and $\alpha_{i}=\alpha_{j}$. From Proposition $4.2, R_{i}^{2 n u}=\left(r_{i, 2} r_{i, 4} \cdots r_{i, 2 n}\right)^{u}$ and so $R_{j}^{2 n}=r_{i, 4} r_{i, 6} \cdots r_{i, 2 n} r_{i, 2}$. Thus $r_{i, 4}$ is the first relation of some $R_{j}^{2 n}$ with $j \in \mathcal{I}$ and $\alpha_{i}=\alpha_{j}$. The result now follows by induction.

Remark. In the above proof, we have also shown that $r_{i, 2}$ is the last relation of $R_{j}^{2 n}$ with $j \in \mathcal{I}$ and $\alpha_{i}=\alpha_{j}$. Thus by induction, each $r_{i, 2 h}$ is also the last relation of some $R_{j}^{2 n}$ with $j \in \mathcal{I}$.

Lemma 4.4. With the notation of Proposition 4.2, let $R_{i}^{2 n}=r_{i, 2} r_{i, 4} \cdots r_{i, 2 n}$ with $i \in \mathcal{I}$. If $r \in \mathcal{R}^{2}$ with $R_{i}^{2 n} r$ in $\mathcal{R}^{2 n+2}$, then $r=r_{i, 2}$.

Proof. Let $g^{2}$ be the basis element in $E(\Lambda)$ with path $r$. Then $g_{i}^{2 n} g^{2} \neq 0$. With the arguments of Lemma 3.1, we have that $z g^{2} \neq 0$ and that there is some $j \in\{1, \ldots, l\}$ with $g_{i}^{2 n} g^{2}=g^{2} g_{j}^{2 n}$. Hence $r$ is the first relation in $R_{i}^{2 n}$, that is, $r=r_{i, 2}$.

Theorem 4.5. With the notation of Proposition 4.2, suppose that $R_{1}^{2 n}=$ $r_{2} r_{4} \cdots r_{2 n}, R_{2}^{2 n}=\tilde{r}_{2} \tilde{r}_{4} \cdots \tilde{r}_{2 n}$ and that $r_{2 n}=\tilde{r}_{2 n}$. Then $R_{1}^{2 n}=R_{2}^{2 n}$. 
Proof. We begin by showing that $r_{2}=\tilde{r}_{2}$. The tails $t\left(R_{1}^{2 n}\right)$ and $t\left(R_{2}^{2 n}\right)$ are both suffixes of $r_{2 n}=\tilde{r}_{2 n}$, so without loss of generality, we may assume that the length of $t\left(R_{1}^{2 n}\right)$ is greater than or equal to the length of $t\left(R_{2}^{2 n}\right)$. Let $\left(r_{2}, r_{3}, \ldots, r_{2 n}\right)$ be the maximal left overlap sequence for $R_{1}^{2 n}$ and let $\left(\tilde{r}_{2}, \tilde{r}_{3}, \ldots, \tilde{r}_{4 n}\right)$ be the maximal left overlap sequence for $\left(R_{2}^{2 n}\right)^{2}$. Note that $\tilde{r}_{2 n+2 i}=\tilde{r}_{2 i}$ for $i=1, \ldots, n$. Now $\tilde{r}_{2 n+1}$ overlaps $t\left(R_{2}^{2 n}\right)$ and so $\tilde{r}_{2 n+1}$ overlaps $t\left(R_{1}^{2 n}\right)$. Since $r_{2 n}=\tilde{r}_{2 n}$, we have that $\left(r_{2}, r_{3}, \ldots, r_{2 n}, \tilde{r}_{2 n+1}, \tilde{r}_{2}\right)$ is a left overlap sequence with path $r_{2} \cdots r_{2 n} \tilde{r}_{2}$. Since this path is tightly packed, it follows from Proposition 2.3 that $R_{1}^{2 n} \tilde{r}_{2} \in \mathcal{R}^{2 n+2}$. Hence from Lemma 4.4 we have $\tilde{r}_{2}=r_{2}$.

Now from the proof of Lemma 4.3 and the remark following, we may repeat this process with the terms $r_{4} r_{6} \cdots r_{2 n} r_{2}$ and $\tilde{r}_{4} \tilde{r}_{6} \cdots \tilde{r}_{2 n} \tilde{r}_{2}$ where $\tilde{r}_{2 n}=r_{2 n}$ and $\tilde{r}_{2}=r_{2}$ to show that $\tilde{r}_{4}=r_{4}$. Hence by induction we have $R_{1}^{2 n}=R_{2}^{2 n}$.

We now show that the coefficients $\alpha_{i}$ for $i \in \mathcal{I}$ are all equal.

Proposition 4.6. Let $z \in Z^{2 n}(E(\Lambda))$ be a non-nilpotent element associated to the equivalence class $\mathcal{E}$. Write $z=\sum_{i \in \mathcal{I}} \alpha_{i} g_{i}^{2 n}+y$ where $y$ is nilpotent, $\alpha_{i} \in K \backslash\{0\}$ and all $R_{i}^{2 n}$ lie in $\mathcal{E}$. Then all the coefficients $\alpha_{i}$ for $i \in \mathcal{I}$ are equal, so $z=$ $\alpha \sum_{i \in \mathcal{I}} g_{i}^{2 n}+y$ for some $\alpha \in K \backslash\{0\}$ where $y$ is nilpotent. Moreover each path $R_{i}^{2 n}$ with $i \in \mathcal{I}$ has the same length.

Proof. With appropriate relabeling of the set $\mathcal{G}^{2 n}$, let $1 \in \mathcal{I}$, and write $R_{1}^{2 n u}=$ $R_{1}^{2 n u-1} t\left(R_{1}^{2 n u}\right)$. Let $t_{1}=t\left(R_{1}^{2 n u}\right)$. By definition of the set $\mathcal{I}$, we have that $\left(g_{1}^{2 n}\right)^{k} g_{1}^{2 n u} \neq 0$ so that $R_{1}^{2 n k} R_{1}^{2 n u}=R_{1}^{2 n k} R_{1}^{2 n u-1} t_{1}$ for all $k \geq 1$.

We show first that $\left(g_{1}^{2 n}\right)^{k} g_{1}^{2 n u-1} \neq 0$ for each $k \geq 1$. Now $\left(g_{1}^{2 n}\right)^{k} g_{1}^{2 n u}=g_{1}^{2 n(k+u)}$ so that

$$
\begin{aligned}
R_{1}^{2 n(k+u)} & =R_{1}^{2 n(k+u)-1} t\left(R_{1}^{2 n(k+u)}\right) \\
& =R_{1}^{2 n(k+u)-1} t\left(R_{1}^{2 n u}\right) \\
& =R_{1}^{2 n(k+u)-1} t_{1}
\end{aligned}
$$

with the penultimate equality coming from the definition of $u$. Thus $R_{1}^{2 n(k+u)-1} t_{1}=$ $R_{1}^{2 n(k+u)}=R_{1}^{2 n k} R_{1}^{2 n u-1} t_{1}$. Hence $R_{1}^{2 n(k+u)-1}=R_{1}^{2 n k} R_{1}^{2 n u-1}$ so that

$$
R_{1}^{2 n k} R_{1}^{2 n u-1} \in \mathcal{R}^{2 n(u+k)-1} .
$$

Thus $\left(g_{1}^{2 n}\right)^{k} g_{1}^{2 n u-1} \neq 0$ for each $k \geq 1$.

Now with $k=1, z \in Z_{\mathrm{gr}}(E(\Lambda))$ so, using the arguments of Lemma 3.1, we have $z g_{1}^{2 n u-1} \neq 0$ and, again relabeling $\mathcal{G}^{2 n}$ if necessary, $g_{1}^{2 n} g_{1}^{2 n u-1}=g_{1}^{2 n u-1} g_{2}^{2 n}$ and $\alpha_{1}=\alpha_{2}$. So $g_{1}^{2 n}$ and $g_{2}^{2 n}$ occur in $z$ with the same coefficients and $\ell\left(R_{1}^{2 n}\right)=\ell\left(R_{2}^{2 n}\right)$.

We show next that $2 \in \mathcal{I}$, that is, $g_{2}^{2 n}$ is not nilpotent. For each $k \geq 1, z^{k} \in$ $Z_{\mathrm{gr}}(E(\Lambda))$ so, using the arguments of Lemma 3.1 , we have $z^{k} g_{1}^{2 n u-1} \neq 0$ and there is some $j(k)$ with $\left(g_{1}^{2 n}\right)^{k} g_{1}^{2 n u-1}=g_{1}^{2 n u-1}\left(g_{j(k)}^{2 n}\right)^{k}$. But $g_{1}^{2 n} g_{1}^{2 n u-1}=g_{1}^{2 n u-1} g_{2}^{2 n}$, so by uniqueness of paths it follows that $j(k)=2$ for all $k$. Hence

$$
\left(g_{1}^{2 n}\right)^{k} g_{1}^{2 n u-1}=g_{1}^{2 n u-1}\left(g_{2}^{2 n}\right)^{k} \text { for all } k \geq 1 .
$$

Thus $g_{1}^{2 n u-1}\left(g_{2}^{2 n}\right)^{k} \neq 0$ and hence $\left(g_{2}^{2 n}\right)^{k} \neq 0$ for all $k \geq 1$. Thus $2 \in \mathcal{I}$.

Now we show that in fact $R_{2}^{2 n u}=t_{1} R_{1}^{2 n u-1}$. With $k=u,\left(g_{1}^{2 n}\right)^{u} g_{1}^{2 n u-1}=$ $g_{1}^{2 n u-1}\left(g_{2}^{2 n}\right)^{u}$ so that

$$
R_{1}^{2 n u} R_{1}^{2 n u-1}=R_{1}^{2 n u-1} R_{2}^{2 n u} .
$$


Write $R_{2}^{2 n u}=q R_{2}^{2 n u-1}$ for some path $q$. Then we have

$$
R_{1}^{2 n u} R_{1}^{2 n u-1}=R_{1}^{2 n u-1} q R_{2}^{2 n u-1} .
$$

By uniqueness of paths, $R_{1}^{2 n u-1}=R_{2}^{2 n u-1}$ and $R_{1}^{2 n u}=R_{1}^{2 n u-1} q$. Since $R_{1}^{2 n u}=$ $R_{1}^{2 n u-1} t_{1}$ it follows that $t_{1}=q$. Thus $R_{2}^{2 n u}=t_{1} R_{1}^{2 n u-1}$.

So, proceeding inductively, we have

$$
\begin{aligned}
R_{1}^{2 n u} & =R_{1}^{2 n u-1} t_{1}=R_{2}^{2 n u-1} t_{2} \\
R_{2}^{2 n u} & =t_{1} R_{1}^{2 n u-1}=R_{3}^{2 n u-1} t_{3} \\
R_{3}^{2 n u} & =t_{2} R_{2}^{2 n u-1} \\
& \vdots \\
R_{f}^{2 n u} & =t_{f-1} R_{f-1}^{2 n u-1}=R_{f}^{2 n u-1} t_{f}
\end{aligned}
$$

with $\{1, \ldots, f\} \subseteq \mathcal{I}$. Moreover $\alpha_{1}=\alpha_{2}=\cdots=\alpha_{f}$ and $\ell\left(R_{1}^{2 n}\right)=\ell\left(R_{2}^{2 n}\right)=\cdots=$ $\ell\left(R_{f}^{2 n}\right)$.

Since the set $I$ is finite, there exists a minimal $f$ where this list of $R_{i}^{2 n u}$ repeats, that is, there exists a minimal $f$ such that $R_{f+1}^{2 n u}=R_{s}^{2 n u}$ for some $1 \leq s \leq f$. We show that $s=1$. Suppose for contradiction that $s>1$. Then $t_{f} R_{f}^{2 n u-1}=R_{f+1}^{2 n u}=$ $R_{s}^{2 n u}=t_{s-1} R_{s-1}^{2 n u-1}$. So by Proposition 1.11, it follows that $R_{f}^{2 n u-1}=R_{s-1}^{2 n u-1}$ and thus $t_{f}=t_{s-1}$. Hence $R_{f}^{2 n u}=R_{f}^{2 n u-1} t_{f}=R_{s-1}^{2 n u-1} t_{s-1}=R_{s-1}^{2 n u}$, which contradicts the minimality of $s$. Thus $s=1$, and so $R_{f+1}^{2 n u}=R_{1}^{2 n u}$.

We have that $\alpha_{1}=\cdots=\alpha_{f}$ so write $\alpha=\alpha_{1}$. Then $g_{1}^{2 n}, \ldots, g_{f}^{2 n}$ all occur in $z$ with coefficient $\alpha$. Moreover from Lemma 4.3, for each relation $r_{i, 2 h}$ in some $R_{1}^{2 n}, \ldots, R_{f}^{2 n}$ there is some $R_{j}^{2 n}$ with $j \in \mathcal{I}$ where $g_{j}^{2 n}$ occurs in $z$ with the same coefficient $\alpha$. We now let $\mathcal{J}_{1}$ denote the subset of $\mathcal{I}$ consisting of all $i$ such that $g_{i}^{2 n}$ occurs in $z$ with coefficient $\alpha$. Continuing in this way, we partition the set $\mathcal{I}$ into disjoint sets $\mathcal{J}_{1}, \ldots, \mathcal{J}_{d}$ with respect to this construction.

Then $z=\sum_{j=1}^{d} \alpha_{j}\left(\sum_{i \in \mathcal{J}_{j}} g_{i}^{2 n}\right)+y$, where $y$ is nilpotent. Let $w_{j}=\sum_{i \in \mathcal{J}_{j}} g_{i}^{2 n}$ so that $z=\sum_{j=1}^{d} \alpha_{j} w_{j}+y$ with $y$ nilpotent and $\alpha_{j} \in K \backslash\{0\}$.

We complete the proof once we have shown that $d=1$, since then $z=$ $\alpha \sum_{i \in \mathcal{I}} g_{i}^{2 n}+y$, where $y$ is nilpotent and $\alpha \in K \backslash\{0\}$. To do this we require the following two propositions; the proof that $d=1$ is the final part of the second proposition.

The next result describes how the different $R^{2 n}$ are related for $g^{2 n}$ occurring in $z$.

Proposition 4.7. Let $i \in \mathcal{I}$. With the notation of Proposition 4.2

$$
R_{i}^{2 n u}=a_{i} r_{i+1,4} r_{i+1,6} \cdots r_{i+1,2 n u} t_{i}
$$

and

$$
R_{i}^{2 n u}=t_{i-1} r_{i-1,2} r_{i-1,4} \cdots r_{i-1,2 n u-2} \tilde{a}_{i-1}
$$

for paths $a_{i}$ and $\tilde{a}_{i}$ with $t_{i} a_{i}=r_{i+1,2}$ and $\tilde{a}_{i-1} t_{i-1}=r_{i-1,2 n u}$.

Proof. Without loss of generality, consider the case $i=1$.

Recall that $R_{2}^{2 n u}=t_{1} R_{1}^{2 n u-1}$ and $R_{2}^{2 n u}=r_{2,2} r_{2,4} \cdots r_{2,2 n u}$ so that $R_{1}^{2 n-1}=$ $a_{1} r_{2,4} r_{2,6} \cdots r_{2,2 n u}$ for some path $a_{1}$ with $t_{1} a_{1}=r_{2,2}$. Now $R_{1}^{2 n u}=R_{1}^{2 n u-1} t_{1}$. Thus $R_{1}^{2 n u}=a_{1} r_{2,4} r_{2,6} \cdots r_{2,2 n u} t_{1}$. 
Similarly using $R_{f}^{2 n u}=R_{f}^{2 n u-1} t_{f}$ and $R_{f}^{2 n u}=r_{f, 2} r_{f, 4} \cdots r_{f, 2 n u}$ we have $R_{f}^{2 n u-1}=$ $r_{f, 2} r_{f, 4} \cdots r_{f, 2 n u-2} \tilde{a}_{f}$ for some path $\tilde{a}_{f}$ with $\tilde{a}_{f} t_{f}=r_{f, 2 n u}$. Now $R_{1}^{2 n u}=R_{f+1}^{2 n u}=$ $t_{f} R_{f}^{2 n u-1}$. Thus $R_{1}^{2 n u}=t_{f} r_{f, 2} r_{f, 4} \cdots r_{f, 2 n u-2} \tilde{a}_{f}$.

The next result is the last part of the proof of Proposition 4.6.

Proposition 4.8. With the notation already introduced, let $p=s_{2} s_{4} \cdots s_{2 n}$ be a tightly packed closed walk in the equivalence class $\mathcal{E}$, with $s_{2}, s_{4}, \ldots, s_{2 n} \in \mathcal{R}^{2}$. Then there is some $j \in \mathcal{J}_{1}$ such that $p=R_{j}^{2 n}$ and, in particular, $p$ is in $\mathcal{R}^{2 n}$. Hence $d=1$.

Proof. With appropriate relabeling of the set $\mathcal{G}^{2 n}$, suppose that $1 \in \mathcal{J}_{1}$ and write $R_{1}^{2 n}=r_{1,2} \ldots r_{1,2 n}$. Consider first all tightly packed closed walks $p=s_{2} s_{4} \cdots s_{2 n}$ which are in the equivalence class $\mathcal{E}$ and whose first relation $s_{2}$ either is $r_{1,2}$ or overlaps $r_{1,2}$. Label these paths $R^{2 n}[1], R^{2 n}[2], \ldots, R^{2 n}[k]$ such that

(1) $R^{2 n}[1]=R_{1}^{2 n}$, and

(2) $r[i+1]_{2}$ overlaps $r[i]_{2}$, for $i=1, \ldots, k-1$, where we write $R^{2 n}[i]=$ $r[i]_{2} r[i]_{4} \cdots r[i]_{2 n}$.

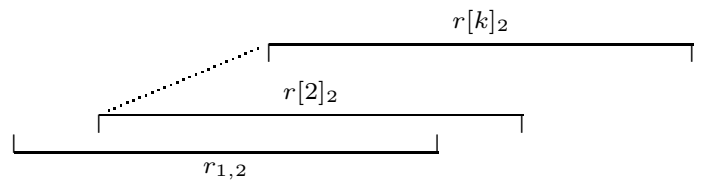

Let $\left(r_{1,2}, r_{1,3}, \ldots, r_{1,2 n u}\right)$ be the maximal left overlap sequence for $\left(R_{1}^{2 n}\right)^{u}$. Then there is a left overlap sequence $\left(r[i]_{2}, r_{1,4}, r[i]_{4}, r_{1,6}, \ldots, r[i]_{2 n}\right)$ with path $R^{2 n}[i]$ since $R_{1}^{2 n}$ is tightly packed. So by Proposition 2.3 , the path $R^{2 n}[i]$ is in $\mathcal{R}^{2 n}$ for $i=1, \ldots, k$.

We now show that $R^{2 n}[2]=R_{j}^{2 n}$ for some $j \in \mathcal{J}_{1}$. To ease notation, let $r[2]_{2 i}=$ $\tilde{r}_{2 i}$ for $i=1, \ldots, n$. If $\tilde{r}_{2}=r_{1,2}$ then $R^{2 n}[2]=R_{1}^{2 n}$ and we are done. Thus suppose $\tilde{r}_{2} \neq r_{1,2}$. There are two cases to consider.

First we suppose that $\tilde{r}_{2 i}=r_{1,2 n l+2 i+1}$ for some $i, l$ with $1 \leq i \leq n-1$ and $1 \leq l \leq u-1$. Since $R_{1}^{2 n}$ is tightly packed, the relation $\tilde{r}_{2 i}$ is situated as follows.

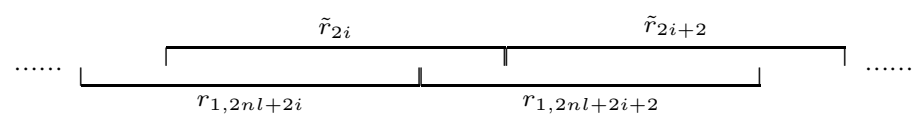

Now $R^{2 n}[2]$ is also tightly packed, so that $\tilde{r}_{2 i+2}=r_{1,2 n l+2 i+3}$. Continuing in this way we have that the maximal left overlap sequence for $\left(R_{1}^{2 n}\right)^{u}=R_{1}^{2 n u}$ has the form

$$
\left(r_{1,2}, r_{1,3}, \ldots, r_{1,2 n l+2 i}, \tilde{r}_{2 i}, r_{1,2 n l+2 i+2}, \tilde{r}_{2 i+2}, \ldots, \tilde{r}_{2 n(u-l)-2}, r_{1,2 n u}\right) .
$$

Now,

$$
\left(r_{1,2}, r_{1,3}, \ldots, r_{1,2 n l+2 i}, \tilde{r}_{2 i}, r_{1,2 n l+2 i+2}, \tilde{r}_{2 i+2}, \ldots, \tilde{r}_{2 n(u-l)-2}\right)
$$

is a maximal left overlap sequence for $R_{1}^{2 n u-1}$. By choice of $u, R_{1}^{2 n u}=R_{1}^{2 n u-1} t_{1}$, and so $t_{1}$ is the path indicated below.

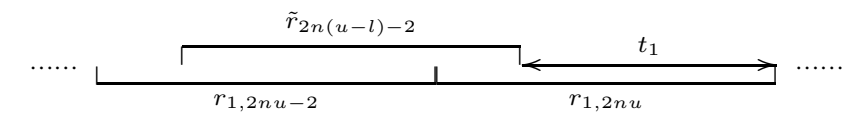


From Proposition 4.7, we have

$$
R_{1}^{2 n u}=a_{1} r_{2,4} r_{2,6} \cdots r_{2,2 n u} t_{1}
$$

and so $\tilde{r}_{2 n(u-l)-2}=r_{2,2 n u}$. Moreover, by definition of the set $\mathcal{J}_{1}$ we have that $2 \in \mathcal{J}_{1}$. Thus $r_{2,2}=r_{2,2 n u+2}=\tilde{r}_{2 n(u-l)}$. Now $R^{2 n}[2]$ is tightly packed and so $\tilde{r}_{2 n(u-l)}=\tilde{r}_{2 n}$. Hence

$$
R_{2}^{2 n u}=\tilde{r}_{2 n} \tilde{r}_{2} \cdots \tilde{r}_{2 n-2} .
$$

Using Lemma 4.3, it follows that $\tilde{r}_{2}$ is the first relation of some $R_{j}^{2 n}$ with $j \in \mathcal{I}$, that is, $R_{j}^{2 n}=\tilde{r}_{2} \tilde{r}_{4} \cdots \tilde{r}_{2 n}$. Again by definition of $\mathcal{J}_{1}$, we have that $j \in \mathcal{J}_{1}$. Thus we have shown that $R^{2 n}[2]=R_{j}^{2 n}$ for some $j \in \mathcal{J}_{1}$.

For the second case, we suppose that $\tilde{r}_{2 i} \neq r_{1,2 n l+2 i+1}$ for all $1 \leq i \leq n-1$ and $1 \leq l \leq u-1$.

From our initial hypothesis, $\tilde{r}_{2}$ overlaps $r_{1,2}$. Now $r_{1,3}$ is the first relation that overlaps $r_{1,2}$ since $\left(r_{1,2}, r_{1,3}\right)$ is a maximal left overlap sequence. Thus $\tilde{r}_{2}$, which is the first relation which starts a tightly packed closed walk and which overlaps $r_{1,2}$, must overlap $r_{1,3}$. This is illustrated in the following diagram.

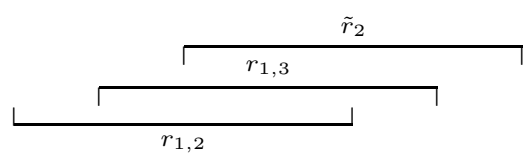

By maximality of the left overlap sequence $\left(r_{2}, r_{3}, \ldots, r_{2 n u}\right)$ for $R_{1}^{2 n u}$, the relation $r_{1,5}$ must begin at or before $\tilde{r}_{4}$. But by the assumptions of this case, $r_{1,5} \neq \tilde{r}_{4}$ and so $\tilde{r}_{4}$ overlaps $r_{1,5}$ and starts strictly after $\mathfrak{o}\left(r_{1,5}\right)$. Continuing in this way, we see that $\tilde{r}_{2 k}$ overlaps $r_{1,2 k+1}$ for $k=1 \ldots, n$. Since $R^{2 n}[2]$ is tightly packed, $\tilde{r}_{2 n l+2 k}=\tilde{r}_{2 k}$ for $k=1 \ldots, n, l=1 \ldots, u-1$. Hence $\tilde{r}_{2 k}$ overlaps $r_{1,2 n l+2 k+1}$ for $k=1 \ldots, n$ and $l=1 \ldots, u-1$.

Now consider the maximal left overlap sequence $\left(r_{1,2}, r_{1,3}, \ldots, r_{1,2 n(u+1)}\right)$ for $\left(R_{1}^{2 n}\right)^{(u+1)}$. As above, we have from Proposition 4.7, that

$$
R_{1}^{2 n u}=a_{1} r_{2,4} r_{2,6} \cdots r_{2,2 n u} t_{1}
$$

and so $r_{1,2 n u-1}=r_{2,2 n u}$. Thus

$$
\left(r_{1,2 n u-1}, r_{1,2 n u+1}, \ldots, r_{1,2 n u+2 n-3}\right)
$$

is a tightly packed path, and hence

$$
\left(r_{1,2 n u+3}, r_{1,2 n u+3}, \ldots, r_{1,2 n u+2 n+1}\right)
$$

is also tightly packed. Thus, since $R^{2 n}$ [2] is tightly packed and $\tilde{r}_{2 n-2}$ overlaps and is not equal to $r_{1,2 n u-1}$, it follows that $\tilde{r}_{2 n}$ overlaps and is not equal to $r_{1,2 n u+1}$. So $\tilde{r}_{2 k}$ overlaps and is not equal to $r_{1,2 n u+2 k+1}$ for $k=1, \ldots, n$. Thus, recalling that $r_{1,2 n u+2 k}=r_{1,2 k}$ for $k=1 \ldots, n$, we have the following diagram.

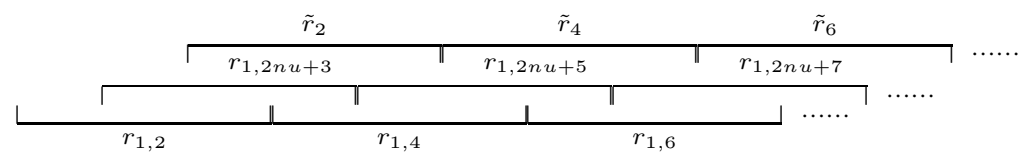

So we have distinct tightly packed sequences

$$
\begin{gathered}
\left(\tilde{r}_{2}, \tilde{r}_{4}, \ldots, \tilde{r}_{2 n}\right), \\
\left(r_{1,2 n u+3}, r_{1,2 n u+5}, \ldots, r_{1,2 n u+2 n+1}\right),
\end{gathered}
$$


and

$$
\left(r_{1,2}, r_{1,4}, \ldots, r_{1,2 n}\right),
$$

for which $\tilde{r}_{2}$ overlaps $r_{1,2 n u+3}$ and $r_{1,2 n u+3}$ overlaps $r_{1,2}$. But this contradicts (2) in the choice of labeling of $R^{2 n}$ [2], and so this case cannot occur.

Thus we have shown that $R^{2 n}[2]=R_{j}^{2 n}$ for some $j \in \mathcal{J}_{1}$. It follows by induction, for each $i=1, \ldots, k$, that $R^{2 n}[i]=R_{j}^{2 n}$ for some $j \in \mathcal{J}_{1}$.

Now let $p=s_{2} s_{4} \cdots s_{2 n}$ be a tightly packed path in the equivalence class $\mathcal{E}$. Then there is some $1 \leq i \leq n$, with either $s_{2}=r_{1,2 i}$ or $s_{2}$ overlaps $r_{1,2 i}$. By Lemma $4.3, r_{1,2 i}$ is the first relation of some $R_{c}^{2 n}$ with $c \in \mathcal{J}_{1}$. We now repeat the above arguments, replacing $R_{1}^{2 n}$ by $R_{c}^{2 n}$, to show that there is some $j \in \mathcal{J}_{1}$ with $p=R_{j}^{2 n}$. Thus, in particular, $p \in \mathcal{R}^{2 n}$.

Finally, suppose for contradiction that $d \geq 2$. Recalling the notation of Proposition 4.6, consider $w_{1}=\sum_{i \in \mathcal{J}_{1}} g_{i}^{2 n}$ and $w_{2}=\sum_{i \in \mathcal{J}_{2}} g_{i}^{2 n}$. By construction, the sets $\mathcal{J}_{1}$ and $\mathcal{J}_{2}$ are disjoint. Let $i \in \mathcal{J}_{2}$. Then $R_{i}^{2 n}$ is a tightly packed closed walk in the equivalence class $\mathcal{E}$. Thus, from above, there is some $j \in \mathcal{J}_{1}$ with $R_{i}^{2 n}=R_{j}^{2 n}$. Hence $i=j$ and the sets $\mathcal{J}_{1}$ and $\mathcal{J}_{2}$ are not disjoint. This gives the required contradiction. Hence $d=1$. This completes the proof.

Thus the proof of Proposition 4.6 is also completed. Hence we may write $z=$ $\alpha \sum_{i \in \mathcal{I}} g_{i}^{2 n}+y$, where $y$ is nilpotent and $\alpha \in K \backslash\{0\}$. Moreover every tightly packed closed walk $s_{2} s_{4} \cdots s_{2 n}$ in the equivalence class $\mathcal{E}$ is some $R_{i}^{2 n}$ with $i \in \mathcal{I}$.

So far we have only discussed non-nilpotent elements in $Z_{\mathrm{gr}}(E(\Lambda))$ of even degree. However, the description of the non-nilpotent elements in $Z_{\mathrm{gr}}(E(\Lambda))$ of odd degree is easily obtained from the above as we now explain.

Let $z=\sum_{i=1}^{l} \alpha_{i} g_{i}^{2 n+1}$ with $\alpha_{i}$ in $K \backslash\{0\}$ for $i=1,2, \ldots, l$ be a non-nilpotent element in $Z_{\mathrm{gr}}(E(\Lambda))$ of odd degree $2 n+1$ (with the characteristic of $K$ necessarily being equal to 2). Observing that the degree of the element was not used in the proofs of Lemma 3.1 and Proposition 3.3, we infer that $z=\sum_{i=1}^{r} z_{i}$ with $z_{i}=$ $\sum_{g_{j}^{2 n+1} \in \mathcal{F}_{i}} \alpha_{j} g_{j}^{2 n+1}$ and each $\mathcal{F}_{i}$ is a subset of $\mathcal{E}_{i}$, and the sets $\mathcal{E}_{i}$ are distinct equivalence classes of closed walks. Moreover, each $z_{i}$ is in $Z_{\mathrm{gr}}(E(\Lambda))$, and $z^{u}=\sum_{i=1}^{r} z_{i}^{u}$ with $z_{i}^{u}=\sum_{g_{j}^{2 n+1} \in \mathcal{F}_{i}} \alpha_{j}^{u}\left(g_{j}^{2 n+1}\right)^{u}$. Suppose now that $z$ is associated to the equivalence class $\mathcal{E}$. Then we can write $z=\sum_{i \in \mathcal{I}} \alpha_{i} g_{i}^{2 n+1}+y$ where $y$ is nilpotent and, for each $i$ in $\mathcal{I}, g_{i}^{2 n+1}$ is non-nilpotent. Then $z^{2}=\sum_{i \in \mathcal{I}} \alpha_{i}^{2}\left(g_{i}^{2 n+1}\right)^{2}+y^{2}$ is also in $Z_{\mathrm{gr}}(E(\Lambda))$ and is non-nilpotent of even degree! It follows from Proposition 4.6 that $z=\alpha \sum_{i \in \mathcal{I}} g_{i}^{2 n+1}+y$ where $\mathcal{I}=\left\{i: R_{i}^{2 n+1}\right.$ is in $\mathcal{E}$ and $g_{i}^{2 n+1}$ is not nilpotent $\}$. Moreover, it is straightforward to see, making the obvious generalisations to the definitions, that $R_{i}^{2 n+1}$ is tightly packed (that is, $R_{i}^{2 n+1}=r_{i, 2} r_{i, 4} \cdots r_{i, 2 n} t_{i}$ ) and is both left and right $2 n$-stable. In addition, there are paths $a_{i}$ and $t_{i}$ such that

$$
R_{i}^{2 n+1}=a_{i} r_{i+1,4} r_{i+1,6} \cdots r_{i+1,2 n} r_{i+1,2}=r_{i, 2} r_{i, 4} \cdots r_{i, 2 n} t_{i}
$$

and

$$
R_{i}^{2 n+1}=t_{i-1} r_{i-1,2} r_{i-1,4} \cdots r_{i-1,2 n}
$$

with $t_{i} a_{i}=r_{i+1,2}$ (in particular $a_{i}=t_{i-1}$ ) and $r_{i-1,2 j}=r_{i+1,2 j+2}$ for $j=$ $1,2, \ldots, n-1$ and $r_{i-1,2 n}=r_{i+1,2}=r_{i, 2 n+1}$.

We now summarise all these results in the following theorem. 
Theorem 4.9. Let $z$ be a homogeneous non-nilpotent element of degree $d$ in $Z_{\mathrm{gr}}(E(\Lambda))$. Then

$$
z=\sum_{i=1}^{r} \alpha_{i} z_{i} \text { with } z_{i} \in Z_{\mathrm{gr}}(E(\Lambda)), \alpha_{i} \in K \backslash\{0\}
$$

where

(i) $\mathcal{E}_{1}, \ldots, \mathcal{E}_{r}$ are distinct equivalence classes of closed walks,

(ii) for each $g^{d}$ occurring in $z_{i}$, the path $R^{d}$ is tightly packed and lies in the equivalence class $\mathcal{E}_{i}$.

We may write

$$
z_{i}=\sum_{j \in \mathcal{I}_{i}} g_{j}^{d}+y_{i}
$$

where $\mathcal{I}_{i}=\left\{j: R_{j}^{d}\right.$ is in $\mathcal{E}_{i}$ and $g_{j}^{d}$ is not nilpotent $\}$, and each $y_{i}$ is nilpotent.

If $d=2 n$, then for each $i=1, \ldots, r$ and each $j \in \mathcal{I}_{i}$, there is some $u \geq 1$ so that $R_{j}^{2 n}$ stabilizes at $u$ with

$$
R_{j}^{2 n u}=a_{j} r_{j+1,4} r_{j+1,6} \cdots r_{j+1,2 n u} t_{j}
$$

and

$$
R_{j}^{2 n u}=t_{j-1} r_{j-1,2} r_{j-1,4} \cdots r_{j-1,2 n u-2} \tilde{a}_{j-1}
$$

for paths $a_{j}$ and $\tilde{a}_{j}$ such that $t_{j} a_{j}=r_{j+1,2}$ and $\tilde{a}_{j-1} t_{j-1}=r_{j-1,2 n u}$.

If $d=2 n+1$, then for each $i, \ldots, r$ and each $j \in \mathcal{I}_{i}$ the path $R_{j}^{2 n+1}$ stabilizes at 1 with

$$
R_{j}^{2 n+1}=a_{j} r_{j+1,4} r_{j+1,6} \cdots r_{j+1,2 n} r_{j+1,2}=r_{j, 2} r_{j, 4} \cdots r_{j, 2 n} t_{j}
$$

and

$$
R_{j}^{2 n+1}=t_{j-1} r_{j-1,2} r_{j-1,4} \cdots r_{j-1,2 n}
$$

with $t_{j} a_{j}=r_{j+1,2}$ (in particular $\left.a_{j}=t_{j-1}\right)$ and $r_{j-1,2 l}=r_{j+1,2 l+2}$ for $l=$ $1,2, \ldots, n-1$ and $r_{j-1,2 n}=r_{j+1,2}=r_{j, 2 n+1}$.

\section{Asymptotic Characterization of the GRAded CEntre modulo NILPOTENTS}

We have seen in the earlier sections that non-nilpotent elements in $Z_{\mathrm{gr}}(E(\Lambda))$ are naturally associated to extending closed walks. For extending closed walks the concept of being stable was introduced. In this section we characterize nonnilpotent elements $z^{u}$ for any non-nilpotent homogeneous element $z$ in $Z_{\mathrm{gr}}(E(\Lambda))$ of even degree and where $u$ is chosen such that the extending paths occurring in $z^{u}$ are stable. We observed in section 2 that $u$ is bounded by $\operatorname{rl}(\Lambda)-1$ (Proposition 2.9).

First we look in more detail at elements of the graded centre. In the next result we consider when nonzero products $g^{m} z \neq 0$ or $z g^{m} \neq 0$ can occur, where $g^{m}$ is in $E(\Lambda)$ and $z \in Z_{\mathrm{gr}}(E(\Lambda))$. Proposition 5.2 then gives more information on the path $R^{m}$ corresponding to such an element $g^{m}$. We keep the notation of the previous section.

Proposition 5.1. With the notation of Theorem 4.9, let $z \in Z^{2 n}(E(\Lambda))$ be nonnilpotent and associated to the equivalence class $\mathcal{E}$. Write $z=\alpha \sum_{i \in \mathcal{I}} g_{i}^{2 n}+y$ where $y=\sum_{i \in \mathcal{I}^{\prime}} g_{i}^{2 n}$ is nilpotent, $\alpha \in K \backslash\{0\}$.

Suppose that $g^{m}$ is in $E(\Lambda)$ such that $g^{m} z \neq 0$ or $z g^{m} \neq 0$. Then $R^{m}$ lies on every representative of $\mathcal{E}$. 
Proof. Suppose that $g^{m} z \neq 0$. Then, following Lemma 3.1, there are $i, j$ with $g^{m} g_{i}^{2 n}=g_{j}^{2 n} g^{m} \neq 0$ and so $R^{m} R_{i}^{2 n}=R_{j}^{2 n} R^{m}$. From Lemma $1.15, R^{m}$ is a subpath of some power of $R_{i}^{2 n}$, and so $R^{m}$ lies on $R_{i}^{2 n}$. By the Remark following Definition 3.2 , we have that $R^{m}$ lies on every representative of $\mathcal{E}$.

The proof that, if $z g^{m} \neq 0$ then $g^{m}$ lies on every representative of $\mathcal{E}$, is similar.

We shall use tightly packed extending closed walks $R^{2 n}$ to construct elements in $Z_{\mathrm{gr}}(E(\Lambda))$. To this end we need to know how these paths interact with paths $R^{m}$ in $\mathcal{R}^{m}$ lying on $R^{2 n}$.

Proposition 5.2. Suppose that $R^{2 n}=r_{2} r_{4} \cdots r_{2 n}$ is a tightly packed, extending closed walk, and that $R^{m} \in \mathcal{R}^{m}$ with $R^{m}$ lying on $R^{2 n}$.

(1) Suppose $\left(R^{2 n}\right)^{s} R^{m} \in \mathcal{R}^{2 n s+m}$ with $s \geq 1$.

(a) If $m$ is even then $R^{m}=\left(R^{2 n}\right)^{c} r_{2} r_{4} \cdots r_{2 i}$ for some $c \geq 0$ and $0 \leq i<$ $n$.

(b) If $m$ is odd then $R^{m}=\left(R^{2 n}\right)^{c} r_{2} r_{4} \cdots r_{2 i} p$ for some $c \geq 0,0 \leq i<n$ and path $p$ such that

$$
p \cdot t\left(\left(R^{2 n}\right)^{s+c} r_{2} r_{4} \cdots r_{2 i+2}\right)=r_{2 i+2} .
$$

(2) Suppose $R^{m}\left(R^{2 n}\right)^{s} \in \mathcal{R}^{2 n s+m}$ with $s \geq 1$.

(a) If $m$ is even then $R^{m}=r_{2 i} r_{2 i+2} \cdots r_{2 n}\left(R^{2 n}\right)^{c}$ for some $c \geq 0$ and $0 \leq i<n$.

(b) If $m$ is odd then $R^{m}=p r_{2 i} r_{2 i+2} \cdots r_{2 n}\left(R^{2 n}\right)^{c}$ for some $c \geq 0,0 \leq i<$ $n$ and path $p$ such that

$$
b\left(r_{2 i-2} r_{2 i} \cdots r_{2 n}\left(R^{2 n}\right)^{s+c}\right) \cdot p=r_{2 i-2} .
$$

Note that if $m$ even and $i=0$ then $R^{m}=\left(R^{2 n}\right)^{c}$, and if $m$ odd and $i=0$ then $R^{m}=\left(R^{2 n}\right)^{c} p$, respectively $R^{m}=p\left(R^{2 n}\right)^{c}$.

Proof. We prove part (1) and leave part (2) to the reader. If $m$ is even, since $R^{2 n}$ is tightly packed and $R^{m}$ lies on $R^{2 n}$, the result easily follows. So suppose that $m$ is odd; again it is clear that $R^{m}=\left(R^{2 n}\right)^{c} r_{2} r_{4} \cdots r_{2 i} p$ for some path $p$. Now $\left(R^{2 n}\right)^{s+c} r_{2} r_{4} \cdots r_{2 i+2}$ is a prefix of $\left(R^{2 n}\right)^{s+c+1}$ which is in $\mathcal{R}^{2 n(s+c+1)}$, so by Corollary 2.4 we have that $\left(R^{2 n}\right)^{s+c} r_{2} r_{4} \cdots r_{2 i+2} \in \mathcal{R}^{2 n(s+c)+2 i+2}$. Let $R_{1}=$ $\left(R^{2 n}\right)^{s+c} r_{2} r_{4} \ldots r_{2 i+2}$ so that $R_{1}=\left(R^{2 n}\right)^{s} R^{m} \cdot t\left(R_{1}\right)$. Thus $p \cdot t\left(R_{1}\right)=r_{2 i+2}$ as required.

As noted before, multiplying elements in $E(\Lambda)$ uses knowledge about the tails and the beginnings of paths. In the next definition, we associate not only one tail but a set of tails to an extending closed walk.

Definition 5.3. Let $R^{2 n}$ be an extending closed walk. The tail set of $R^{2 n}$ is the set of paths $p_{i}$ for $0 \leq i<2 n$, such that if $\left(R^{2 n}\right)^{2}=R^{2 n+i} q$ with $R^{2 n+i} \in \mathcal{R}^{2 n+i}$ then $p_{i}=t\left(R^{2 n+i}\right)$, that is, $p_{i}$ is the tail path of $R^{2 n+i}$.

The beginning set of $R^{2 n}$ is the set of paths $p_{i}$ for $0 \leq i<2 n$, such that if $\left(R^{2 n}\right)^{2}=q R^{2 n+i}$ with $R^{2 n+i} \in \mathcal{R}^{2 n+i}$ then $p_{i}=b\left(R^{2 n+i}\right)$, that is, $p_{i}$ is the beginning path of $R^{2 n+i}$.

In the next definition we look at maximal overlaps of a path with a relation. This is motivated by Definition 1.5, and uses Definition 1.1, which we recall does not require the paths $P$ and $Q$ to be in $\mathcal{R}^{2}$. 
Definition 5.4. Let $p$ be a path in $K \mathcal{Q}$ and let $r$ be in $\mathcal{R}^{2}$.

(1) The relation $r$ maximally left overlaps the path $p$ if there are paths $U, V \in$ $K \mathcal{Q}$ with

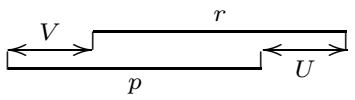

such that

(i) $p U=V r$, and

(ii) $V r \neq P R Q$ for all non-trivial paths $Q$ and all $R \in \mathcal{R}^{2}$.

(Note that $V$ may be a trivial path.)

(2) The path $p$ maximally right overlaps the relation $r$ if there are paths $U, V \in$ $K \mathcal{Q}$ with

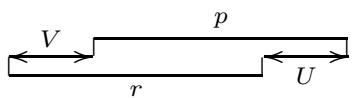

such that

(i) $r U=V p$, and

(ii) $r U \neq P R Q$ for all non-trivial paths $P$ and all $R \in \mathcal{R}^{2}$.

(Note that $U$ may be a trivial path.)

The next result uses the above definitions to describe the relations which maximally overlap a tail or a beginning path of some $R_{i}^{2 n u}$ where $g_{i}^{2 n}$ occurs in a non-nilpotent element of $Z_{\mathrm{gr}}(E(\Lambda))$.

Proposition 5.5. With the notation of Theorem 4.9, let $z \in Z^{2 n}(E(\Lambda))$ be nonnilpotent and associated to the equivalence class $\mathcal{E}$. Write $z=\alpha \sum_{i \in \mathcal{I}} g_{i}^{2 n}+y$ with $y$ nilpotent and $\alpha \in K \backslash\{0\}$.

Let $r \in \mathcal{R}^{2}$ be a relation such that, for some $i \in \mathcal{I}$, either $r$ maximally left overlaps some path $p$ in the tail set of $R_{i}^{2 n u}$, or some path $p$ in the beginning set of $R_{i}^{2 n u}$ maximally right overlaps the relation $r$. Then $r$ lies on every representative of $\mathcal{E}$.

Proof. Suppose the relation $r$ maximally left overlaps $p$ where $p$ is in the tail set of $R_{i}^{2 n u}$. Let $j$ be such that $0 \leq j<2 n u,\left(R_{i}^{2 n u}\right)^{2}=R^{2 n u+j} q$ for some path $q$ with $R^{2 n u+j} \in \mathcal{R}^{2 n u+j}$, and $p=t\left(R^{2 n u+j}\right)$. Let $\left(r_{2}, r_{3}, \ldots, r_{2 n u+j}\right)$ be the maximal left overlap sequence for $R^{2 n u+j}$. Since $r$ maximally left overlaps $p$ and $p=t\left(R^{2 n u+j}\right)$, we see that $S=\left(r_{2}, r_{3}, \ldots, r_{2 n u+j}, r\right)$ is a maximal left overlap sequence.

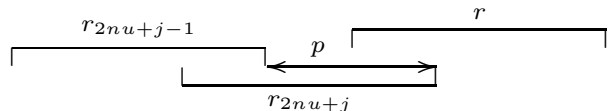

Let $R^{2 n u+j+1}$ be the element in $\mathcal{R}^{2 n u+j+1}$ corresponding to $S$, and let $g^{2 n u+j+1}$ be the corresponding basis element of $E(\Lambda)$. Then $R_{i}^{2 n u} R^{2 n u+j+1} \in \mathcal{R}^{4 n u+j+1}$ so that $g_{i}^{2 n u} g^{2 n u+j+1} \neq 0$ and hence $z^{u} g^{2 n u+j+1} \neq 0$. By Proposition 5.1, the path $R^{2 n u+j+1}$ lies on every representative of $\mathcal{E}$. It follows that the last relation in $R^{2 n u+j+1}$, namely $r$, lies on every representative of $\mathcal{E}$.

The other case is similar and is left to the reader.

Definition 5.6. A tightly packed path $r_{2} r_{4} \cdots r_{2 n}$ with $r_{2}, r_{4}, \ldots, r_{2 n} \in \mathcal{R}^{2}$ is said to be relation simple if, whenever $i \neq j$, then $r_{2 i} \neq r_{2 j}$. 
This definition enables us to describe the minimal closed walks which in some sense generate a non-nilpotent element in $Z_{\mathrm{gr}}(E(\Lambda))$.

Proposition 5.7. With the notation of Theorem 4.9, let $z \in Z^{2 n}(E(\Lambda))$ be nonnilpotent and associated to the equivalence class $\mathcal{E}$. Write $z=\alpha \sum_{i \in \mathcal{I}} g_{i}^{2 n}+y$ with $y$ nilpotent and $\alpha \in K \backslash\{0\}$.

Then, for each $i \in \mathcal{I}$, there is a relation simple, closed walk $W_{i}$ such that $R_{i}^{2 n}=$ $W_{i}^{c_{i}}$ for some $c_{i} \geq 1$. Moreover each $W_{i}$ lies on every representative of $\mathcal{E}$.

Proof. Let $i \in \mathcal{I}$ and write $R^{2 n}$ for $R_{i}^{2 n}$. If $R^{2 n}$ is relation simple then we are done. So suppose that $R^{2 n}$ is not relation simple. Let $R^{2 n}=r_{2} r_{4} \cdots r_{2 n}$. Let $k \leq n$ be the smallest integer such that there is some $j$, with $1 \leq k<j \leq n$, and $r_{2 k}=r_{2 j}$.

Suppose for contradiction that $k>1$. Then

$$
r_{2 j} r_{2 j+2} \cdots r_{2 n} r_{2} \cdots r_{2 j-2} \neq r_{2 k} r_{2 k+2} \cdots r_{2 n} r_{2} \cdots r_{2 k-2}
$$

since if

$$
r_{2 j} r_{2 j+2} \cdots r_{2 n} r_{2} \cdots r_{2 j-2}=r_{2 k} r_{2 k+2} \cdots r_{2 n} r_{2} \cdots r_{2 k-2}
$$

then the $r_{2}$ on the left hand side must equal $r_{2 k-2 j+2 n+2}$ which contradicts $k>1$. So writing $R_{j}^{2 n}=r_{2 j} r_{2 j+2} \cdots r_{2 n} r_{2} \cdots r_{2 j-2}$ and $R_{k}^{2 n}=r_{2 k} r_{2 k+2} \cdots r_{2 n} r_{2} \cdots r_{2 k-2}$, we have that $R_{k}^{2 n} \neq R_{j}^{2 n}$. By Proposition 4.8 , we have $k, j \in \mathcal{I}$ and so $g_{k}^{2 n}$ and $g_{j}^{2 n}$ are non-nilpotent. Since $r_{2 j}=r_{2 k}$ and using the remark following Lemma 4.3, this gives a contradiction to Theorem 4.5. Hence $k=1$.

Choose $j$ minimal such that $1<j \leq n$ and $r_{2 j}=r_{2}$. Now $R_{j}^{2 n}$ and $R^{2 n}$ both have first relation $r_{2}$ so, again using Theorem 4.5, we have that $R_{j}^{2 n}=R^{2 n}$. Let $W=r_{2} r_{4} \cdots r_{2 j-2}$. Then $R^{2 n}=W^{c}$ or $R^{2 n}=W^{c} r_{2} r_{4} \cdots r_{2 s}$ for some $c \geq 1$ and $1<s<j-1$. We show that the second case cannot occur. For, suppose that $R^{2 n}=W^{c} r_{2} r_{4} \cdots r_{2 s}$. Then, since $R^{2 n}$ is extending, we have $r_{2 s+2}=r_{2}$. By minimality of $j$ it follows that $2 j \leq 2 s+2$ and hence $s \geq j-1$. But this contradicts the choice of $s$. Therefore this case does not occur and $R^{2 n}=W^{c}$.

It is now immediate from Definition 3.2 that $W$ and $R^{2 n}$ are in the same equivalence class, and hence $W$ lies on every representative of the equivalence class $\mathcal{E}$. Finally, by similar arguments to those given above, it follows that $W$ is relation simple.

A tightly packed, extending walk $R^{2 n}$ need not in general be such that the relations $r_{3}, r_{5}, \ldots, r_{2 n-1}$ of the maximal left overlap sequence form a tighly packed path $r_{3} r_{5} \cdots r_{2 n-1}$, but we show that this is asymptotically the case for the $R^{2 n}$ occurring in non-nilpotent elements in $Z_{\mathrm{gr}}(E(\Lambda))$. First we make a precise definition describing the above phenomenon.

Definition 5.8. A tightly packed, extending walk $R^{2 n}$ is said to be tightly covered if there is some $s \geq 1$ such that

(i) if $\left(r_{2}, r_{3}, \ldots, r_{2 n s}\right)$ is the maximal left overlap sequence of $\left(R^{2 n}\right)^{s}$ then $r_{2 n(s-1)-1} r_{2 n(s-1)+1} \cdots r_{2 n s-3}$ is a tightly packed walk, and

(ii) if $\left(\tilde{r}_{2}, \tilde{r}_{3}, \ldots, \tilde{r}_{2 n s}\right)$ is the maximal right overlap sequence of $\left(R^{2 n}\right)^{s}$ then $\tilde{r}_{3} \tilde{r}_{5} \cdots \tilde{r}_{2 n+1}$ is a tightly packed walk.

The next result connects this notion to that of being stable.

Proposition 5.9. Suppose that $R^{2 n}$ is a tightly covered closed walk with $t\left(R^{2 n u}\right)=$ $t\left(R^{2 n(u+1)}\right)$ and $b\left(R^{2 n u}\right)=b\left(R^{2 n(u+1)}\right)$. 
(1) If $\left(r_{2}, r_{3}, \ldots, r_{2 n(u+1)}\right)$ is the maximal left overlap sequence for $R^{2 n(u+1)}$ then $r_{2 n u-1} r_{2 n u+1} \cdots r_{2 n(u+1)-3}$ is a tightly packed, extending walk.

(2) If $\left(\tilde{r}_{2}, \tilde{r}_{3}, \cdots \tilde{r}_{2 n(u+1)}\right)$ is the maximal right overlap sequence for $R^{2 n(u+1)}$ then $\tilde{r}_{3} \tilde{r}_{5} \cdots \tilde{r}_{2 n+1}$ is a tightly packed, extending walk.

Proof. (1) We have $t\left(R^{2 n u}\right)=t\left(R^{2 n(u+1)}\right)$ and $r_{2 n u}=r_{2 n(u+1)}$ so that $r_{2 n u-1}=$ $r_{2 n(u+1)-1}$. Thus $R^{2 n}$ is left $(2 n u-1)$-stable. Hence, by Proposition 2.11 , for $i \geq$ $2 n u-1$, we have $r_{i}=r_{i+2 n}$. Since $R^{2 n}$ is tightly covered, for sufficiently large $s$, the path $r_{2 n s-1} r_{2 n s+1} \cdots r_{2 n(s+1)-3}$ is tightly packed. But for $s \geq u, r_{2 n s-1}=r_{2 n u-1}$ and $r_{2 n s+j}=r_{2 n u+j}$ for $0 \leq j \leq 2 n$. Thus $r_{2 n u-1} r_{2 n u+1} \cdots r_{2 n(u+1)-3}$ is a tightly packed walk. Moreover $\left(r_{2 n u-1}, r_{2 n u}, \ldots, r_{2 n(u+1)-3}\right)$ is a left overlap sequence, so by Proposition 2.3 , we have that $r_{2 n u-1} r_{2 n u+1} \cdots r_{2 n(u+1)-3}$ is in $\mathcal{R}^{2 n}$. Indeed $r_{2 n(u+1)-1}=r_{2 n u-1}$ and so we have the following left overlap sequence

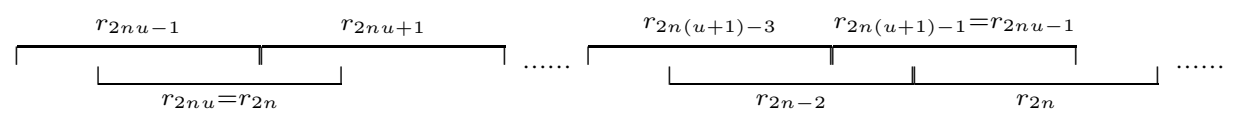

Hence from Proposition 2.3, $r_{2 n u-1} r_{2 n u+1} \cdots r_{2 n(u+1)-3}$ is an extending closed walk. The result now follows.

(2) This is similar to (1).

Consider all relation simple, extending closed walks in the equivalence class $\mathcal{E}$. Since the number of relations in a relation simple walk is bounded above by the total number of (minimal) relations generating $I$, we see that there is a finite number of relation simple walks. Hence the set of relation simple, extending walks in $\mathcal{E}$ is finite. We denote this set by $\Delta_{\mathcal{E}}$.

For each $p \in \Delta_{\mathcal{E}}$, we may write $p$ as a product of $s_{p}$ relations, that is, $p=$ $r_{2} r_{4} \cdots r_{2 s_{p}}$. Let $M$ be the least common multiple of $\left\{s_{p} \mid p \in \Delta_{\mathcal{E}}\right\}$. Then, for each $p$, there is some $m_{p} \geq 1$ such that $p^{m_{p}}$ is the product of $M$ relations.

By Proposition 2.12, for each $p \in \Delta_{\mathcal{E}}$, there is $u_{p} \geq 1$ such that $p^{m_{p}}$ stabilizes at $u_{p}$. Let $u$ be the least common multiple of $\left\{u_{p} \mid p \in \Delta_{\mathcal{E}}\right\}$. Then each $p \in \Delta_{\mathcal{E}}$ stabilizes at $u$ and hence $p^{m_{p} u}$ is a product of $M u$ relations and has the property that $t\left(p^{m_{p} u}\right)=t\left(p^{m_{p}(u+1)}\right)$ and $b\left(p^{m_{p} u}\right)=b\left(p^{m_{p}(u+1)}\right)$. Let $\Delta_{\mathcal{E}}^{u}=\left\{p^{m_{p} u} \mid p \in \Delta_{\mathcal{E}}\right\}$. For each $q \in \Delta_{\mathcal{E}}^{u}$ let $g_{q}$ denote the corresponding element of $\mathcal{G}^{2 N}$.

With all the previous definitions and results, we now characterize non-nilpotent elements of sufficiently high even degree in $Z_{\mathrm{gr}}(E(\Lambda))$.

Theorem 5.10. With the above notation, let $\mathcal{E}$ be an equivalence class of closed walks in $\mathcal{Q}$ and let $W$ be a closed walk in $\mathcal{E}$. Let $N=M u$. Then $\sum_{q \in \Delta_{\mathcal{E}}^{u}} g_{q}$ is in $Z_{\mathrm{gr}}(E(\Lambda))$ if and only if

(1) each $q \in \Delta_{\mathcal{E}}^{u}$ is tightly covered;

(2) all tightly packed, extending walks $r_{2} r_{4} \cdots r_{2 N}$ that lie on $W$ are in $\Delta_{\mathcal{E}}^{u}$;

(3) if a relation $r$ does not lie on $W$ then, for all $q \in \Delta_{\mathcal{E}}^{u}$ and all paths $p$ in the tail set of $q$, the relation $r$ does not maximally left overlap the path $p$;

(4) if a relation $r$ does not lie on $W$ then, for all $q \in \Delta_{\mathcal{E}}^{u}$ and all paths $p$ in the beginning set of $q$, the path $p$ does not maximally right overlap the relation $r$.

Proof. Let $z=\sum_{q \in \Delta_{\mathcal{E}}^{u}} g_{q}$, and suppose that $z \in Z_{\mathrm{gr}}(E(\Lambda))$. We show first that $q$ is tightly covered. Let $\left(r_{2}, r_{3}, \ldots, r_{4 N}\right)$ be the maximal left overlap sequence for $q^{2}$. 
Since $q$ is left $(2 N-1)$-stable, we have the overlap sequence

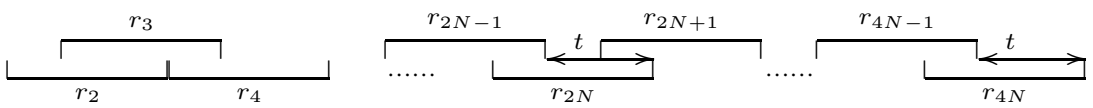

for $q^{2}$ with $r_{2 N-1}=r_{4 N-1}$ and $r_{2 N}=r_{4 N}$. By Proposition 4.7, the path $t r_{2 N+2} r_{2 N+4} \cdots r_{4 N-2} \tilde{a}$ where $\tilde{a} t=r_{4 N}$ is the path $R_{j}^{2 N}$ for some $j$ in $\mathcal{I}$ and so $g_{j}^{2 N}$ occurs in $z$. Thus this path is tightly packed and hence $r_{2 N+1} r_{2 N+3} \cdots r_{4 N-1}$ is tightly packed. But from Proposition 4.7 again, $t$ is the prefix of some relation and so $\mathfrak{t}\left(r_{2 N-1}\right)=\mathfrak{o}\left(r_{2 N+1}\right)$. Hence $r_{2 N-1} r_{2 N+1} \cdots r_{4 N-3}$ is tightly packed. Similarly, since $q$ is right $(2 N-1)$-stable, if $\left(\tilde{r}_{2}, \tilde{r}_{3}, \ldots, \tilde{r}_{4 N}\right)$ is the maximal right overlap sequence for $q^{2}$, then $\tilde{r}_{3} \tilde{r}_{5} \cdots \tilde{r}_{2 N+1}$ is tightly packed. Thus $q$ is tightly covered.

Property (2) follows from Proposition 4.8, and properties (3) and (4) follow from Proposition 5.5.

Now suppose that properties (1)-(4) hold, and let $z=\sum_{q \in \Delta_{\varepsilon}^{u}} g_{q}$. Suppose first that $R^{m} \in \mathcal{R}^{m}$ with $z g^{m} \neq 0$. We show that $z g^{m}=g^{m} z$. First we show that $R^{m}$ lies on $W$. Since $z g^{m} \neq 0$, we have that $q R^{m}$ is in $\mathcal{R}^{2 N+m}$ for some $q \in \Delta_{E}^{u}$. Let $\left(r_{2}, r_{3}, \ldots, r_{2 N+m}\right)$ be the maximal left overlap sequence for $q R^{m}$. Now $q=R^{2 N-1} t(q)$ where $R^{2 N-1} \in \mathcal{R}^{2 N-1}$ has maximal left overlap sequence $\left(r_{2}, r_{3}, \ldots, r_{2 N-1}\right)$ and $r_{2 N+1}$ maximally left overlaps $t(q)$. By hypothesis (3), since $t(q)$ is in the tail set of $q$, we have that $r_{2 N+1}$ lies on $W$. Next, let $q^{*}$ be the path associated to the maximal left overlap sequence $\left(r_{2}, r_{3}, \ldots, r_{2 N+1}\right)$, so that $q^{*}=q t\left(q^{*}\right)$. Noting that $q^{*}$ lies on $W$, the path $t\left(q^{*}\right)$ is in the tail set of $q$, and $r_{2 N+2}$ maximally left overlaps $t\left(q^{*}\right)$. Thus $r_{2 N+2}$ lies on $W$. Since $q$ is left $(2 N-1)$-stable, we may continue inductively showing that $R^{m}$ lies on $W$.

Suppose that $m$ is even. By Proposition 5.2, $R^{m}=q^{c} r_{2} r_{4} \cdots r_{2 i}$. Let $q^{\prime}=$ $r_{2 i+2} \cdots r_{2 N} r_{2} \cdots r_{2 i}$, so that $q^{\prime}$ is a tightly packed, extending closed walk. Hence by (2), $g_{q^{\prime}}$ occurs in $z$. Clearly $R^{m} q^{\prime}=q R^{m}$ as paths. By uniqueness of the corresponding paths, $g^{m} z=g^{m} g_{q^{\prime}}$ and $z g^{m}=g_{q} g^{m}$. Hence $z g^{m}=g^{m} z$.

Now suppose that $m$ is odd. Applying Proposition 5.2, we see that $R^{m}=$ $q^{c} r_{2} r_{4} \cdots r_{2 i} p$ with $p t\left(q^{c+1} r_{2} r_{4} \cdots r_{2 i+2}\right)=r_{2 i+2}$. Since $q R^{m}$ is in $\mathcal{R}^{2 N+m}$, we have that $t\left(q R^{m}\right)=t\left(R^{m}\right)=p$. Recalling that $\left(r_{2}, r_{3}, \ldots, r_{4 N}\right)$ is the maximal left overlap sequence for $q^{2}$, and since $q$ is tightly covered by hypothesis (1), we have from Proposition 5.9 that $r_{2 N-1} r_{2 N+1} \cdots r_{4 N-3}$ is a tightly packed, extending walk. Now $q$ is left $(2 N-1)$-stable so the path $q^{\prime}=r_{2 N+2 i+3} r_{2 N+2 i+5} \cdots r_{4 N+2 i+1}$ is also a tightly packed, extending walk and $\mathfrak{t}\left(r_{2 N+2 i+1}\right)=\mathfrak{t}\left(r_{4 N+2 i+1}\right)=\mathfrak{o}\left(r_{2 N+2 i+3}\right)$. Thus, from hypothesis (2), the element $g_{q^{\prime}}$ occurs in $z$.

We now show that $\mathfrak{t}\left(R^{m}\right)=\mathfrak{o}\left(q^{\prime}\right)$ so that we may consider the path $R^{m} q^{\prime}$. Let $s$ be the last relation in $R^{m}$ so $s$ is placed like this.

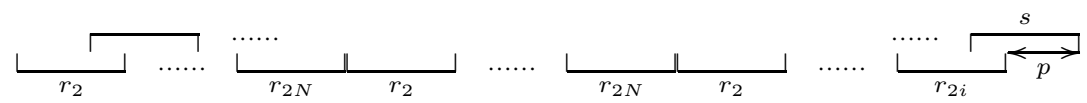

Since $t\left(q R^{m}\right)=t\left(R^{m}\right)$, the relation $s$ is also the last relation in $q R^{m}$, that is, $s=r_{2 N+2 i+1}$, since $q$ is $(2 N-1)$-stable. Hence $\mathfrak{t}\left(R^{m}\right)=\mathfrak{t}(s)=\mathfrak{t}\left(r_{2 N+2 i+1}\right)=$ $\mathfrak{o}\left(r_{2 N+2 i+3}\right)=\mathfrak{o}\left(q^{\prime}\right)$. It is now clear that $R^{m} q^{\prime}=q R^{m}$ since $q^{\prime}$ is tightly packed. So by uniqueness of corresponding paths, it follows that $z g^{m}=g_{q} g^{m}=g^{m} g_{q^{\prime}}=g^{m} z$. Thus if $z g^{m} \neq 0$, then $z g^{m}=g^{m} z$.

Similarly, if $R^{m} \in \mathcal{R}^{m}$ with $g^{m} z \neq 0$ then $g^{m} z=z g^{m}$. Hence $z$ is in $Z_{\mathrm{gr}}(E(\Lambda))$ and this completes the proof. 


\section{Finite GENERATION}

In this section we show that $Z_{\mathrm{gr}}(E(\Lambda)) / \mathcal{N}_{Z}$ is a finitely generated commutative $K$-algebra of Krull dimension at most one. We keep the notation of the previous sections.

We begin by showing there are only a finite number of equivalence classes $\mathcal{E}$ that occur in an element of $Z_{\text {gr }}(E(\Lambda)) / \mathcal{N}_{Z}$.

Proposition 6.1. The number of equivalence classes that can occur in elements of the graded centre is bounded by the number of relations in the minimal generating set $\mathcal{R}^{2}$ that was chosen for the ideal I.

Proof. We first consider elements of even degree. Let $z_{1}$ and $z_{2}$ be non-nilpotent homogeneous elements in $Z_{\mathrm{gr}}(E(\Lambda))$ of degrees $2 n_{1}$ and $2 n_{2}$, respectively. Suppose further that $z_{i}$ is associated to the equivalence class $\mathcal{E}_{i}$. Let $R_{1}^{2 n_{1}}=r_{2} r_{4} \cdots r_{2 n_{1}}$ and $R_{2}^{2 n_{2}}=\tilde{r}_{2} \tilde{r}_{4} \cdots \tilde{r}_{2 n_{2}}$ be paths representing non-nilpotent basis elements in $E(\Lambda)$ occurring in $z_{1}$ and $z_{2}$, respectively. Thus $R_{1}^{2 n_{1}}$ is in $\mathcal{E}_{1}$ and $R_{2}^{2 n_{2}}$ is in $\mathcal{E}_{2}$. Suppose that $r_{2 i}=\tilde{r}_{2 j}$ for some $i$ and $j$. By taking appropriate powers we may assume that $n_{1}=n_{2}=n$ and that both $R_{1}^{2 n}$ and $R_{2}^{2 n}$ are left and right $(2 n-1)$-stable. Applying Lemma 4.3 and Theorem 4.5 we infer that $\mathcal{E}_{1}=\mathcal{E}_{2}$.

If $z$ is a non-nilpotent element in $Z_{\mathrm{gr}}(E(\Lambda))$ of odd degree, then $z^{2}$ is a nonnilpotent element in $Z_{\mathrm{gr}}(E(\Lambda))$ of even degree. Hence we can apply the above argument. It follows from this that the number of different equivalence classes is bounded by the number of elements in $\mathcal{R}^{2}$.

The following class of examples shows that the number of equivalence classes of closed walks associated to homogeneous non-nilpotent elements in the graded centre of the Ext algebra of a monomial algebra can be arbitrary large.

Example 6.2. Choose $n$ pairs of positive integers $\left\{\left(l_{1}, l_{1}^{\prime}\right),\left(l_{2}, l_{2}^{\prime}\right), \ldots,\left(l_{n}, l_{n}^{\prime}\right)\right\}$ where $l_{i}^{\prime} \geq 2$. Consider the quivers $\mathcal{Q}_{i}=\widetilde{\mathbb{A}}_{l_{i}}$ with cyclic orientation. Choose a vertex $v_{1}^{\prime}$ of $\mathcal{Q}_{1}$, two distinct vertices $v_{i}$ and $v_{i}^{\prime}$ of $\mathcal{Q}_{i}$ for $i=2,3, \ldots, n-1$ and finally one vertex $v_{n}$ of $\mathcal{Q}_{n}$. Glue a copy of $\mathbb{A}_{m}$ with some orientation and size $m \geq 1$ between the vertices $v_{i}^{\prime}$ and $v_{i+1}$ for $i=1,2, \ldots, n-1$. Call the resulting quiver $\mathcal{Q}$. Let $\Lambda=K \mathcal{Q} / I$ where $I$ is generated by all paths of length $l_{i}^{\prime}$ in subquivers $\mathcal{Q}_{i}$. Then $\Lambda$ is a monomial algebra with $n$ different equivalence classes of closed walks in the graded centre of $E(\Lambda)$.

Next we show that each equivalence class $\mathcal{E}$ gives rise to at most one basis element in any given degree of $Z_{\mathrm{gr}}(E(\Lambda)) / \mathcal{N}_{Z}$.

Proposition 6.3. Let $\mathcal{E}$ be an equivalence class and let $n$ be a positive integer.

(a) Let $V_{\mathcal{E}}$ be the subspace of $Z^{n}(E(\Lambda)) / \mathcal{N}_{Z}^{n}$ generated by all elements associated to $\mathcal{E}$. Then $\operatorname{dim}_{K} V_{\mathcal{E}} \leq 1$.

(b) Let $r$ be the number of equivalence classes $\mathcal{E}$. Then

$$
\operatorname{dim}_{K} Z^{n}(E(\Lambda)) / \mathcal{N}_{Z}^{n} \leq r
$$

for all $n \geq 1$.

Proof. The claim in (a) is an immediate consequence of Theorem 4.9, and the statement in (b) is a direct consequence of (a). 
Now we apply the above to give further information on the multiplication of elements associated to the same equivalence class $\mathcal{E}$.

Lemma 6.4. Let $z$ and $z^{\prime}$ be two homogeneous non-nilpotent elements in $Z_{\mathrm{gr}}(E(\Lambda))$ associated to the same equivalence class $\mathcal{E}$. Then $z z^{\prime}$ is a homogeneous non-nilpotent element in $Z_{\mathrm{gr}}(E(\Lambda))$.

Proof. Let $z$ and $z^{\prime}$ be two homogeneous non-nilpotent elements in $Z_{\mathrm{gr}}(E(\Lambda))$ associated to the same equivalence class $\mathcal{E}$ of degrees $m$ and $n$, respectively. The elements $z^{n}$ and $\left(z^{\prime}\right)^{m}$ are homogeneous non-nilpotent elements in $Z_{\mathrm{gr}}(E(\Lambda))$ of degree $m n$ so, modulo nilpotence, we have $\left(z^{\prime}\right)^{m}=\alpha z^{n}$ for some $\alpha$ in $K \backslash\{0\}$ by Proposition 6.3(a). Furthermore,

$$
\left(z z^{\prime}\right)^{m i}= \pm z^{m i}\left(z^{\prime}\right)^{m i}= \pm \alpha^{i} z^{(m+n) i} \neq 0
$$

for all $i>0$, so that $z z^{\prime}$ is a non-nilpotent element in $Z_{\mathrm{gr}}(E(\Lambda))$.

Let $\mathcal{E}$ be an equivalence class. Let $D$ be the set of positive integers $n$ such that there is at least one non-nilpotent element $z$ in $Z_{\mathrm{gr}}(E(\Lambda))$ of degree $n$ associated to the equivalence class $\mathcal{E}$. By the above lemma the set $D$ is closed under addition and multiplication by positive integers. Let $D=\left\{n_{i}\right\}_{i=1}^{\infty}$, where $n_{1}<n_{2}<n_{3}<\ldots$.

To prove that there are only a finite number of generators required for $Z_{\mathrm{gr}}(E(\Lambda)) / \mathcal{N}_{Z}$ as a $K$-algebra, we use the following lemma. A proof can be based on the following result of James Joseph Sylvester: Let $a$ and $b$ be positive integers. If $\operatorname{gcd}(a, b)=d$, then $a b-a-b$ is the largest multiple of $d$ which cannot be expressed in the form $a x+b y$ for positive integers $x$ and $y$.

Lemma 6.5. Let $\left\{a_{i}\right\}_{i=1}^{\infty}$ be a subset of $\mathbb{N}$ with $a_{1}<a_{2}<a_{3} \ldots$ For $t \geq 1$, let

$$
D\left(\left\{a_{1}, \ldots, a_{t}\right\}\right)=\left\{\sum_{i=1}^{t} a_{i} b_{i}: b_{i} \geq 0\right\}
$$

and

$$
D\left(\left\{a_{i}\right\}_{i=1}^{\infty}\right)=\bigcup_{t \in \mathbb{N}} D\left(\left\{a_{1}, \ldots, a_{t}\right\}\right) .
$$

Then there exists $N$ such that

$$
D\left(\left\{a_{i}\right\}_{i=1}^{\infty}\right)=D\left(\left\{a_{1}, a_{2}, \ldots, a_{N}\right\}\right) .
$$

We are now ready to give the main result of this section, namely the finite generation of $Z_{\mathrm{gr}}(E(\Lambda)) / \mathcal{N}_{Z}$ as a $K$-algebra.

Theorem 6.6. Let $\Lambda=K \mathcal{Q} / I$ be an indecomposable monomial algebra. Then $Z_{\mathrm{gr}}(E(\Lambda)) / \mathcal{N}_{Z}$ is a finitely generated commutative $K$-algebra of Krull dimension at most one.

Proof. By Theorem 4.9 any homogeneous non-nilpotent element $z$ of degree $n$ in $Z_{\mathrm{gr}}(E(\Lambda))$ can be written as a sum of elements $z_{i}$ in $Z_{\mathrm{gr}}(E(\Lambda))$ of degree $n$, where each $z_{i}$ is associated to a distinct equivalence class $\mathcal{E}_{i}$ for $i=1,2, \ldots, r$. In addition the product of any two elements associated to two different equivalence classes is zero (Proposition 5.1). Hence it is enough to show that each subring of $Z_{\mathrm{gr}}(E(\Lambda)) / \mathcal{N}_{Z}$ generated by elements associated to a fixed equivalence class is a finitely generated $K$-algebra.

Let $\mathcal{E}$ be an equivalence class. Consider again the set $D$ of positive integers $n$ such that there is at least one non-nilpotent element $z$ in $Z_{\mathrm{gr}}(E(\Lambda))$ of degree $n$ 
associated to $\mathcal{E}$. By Lemma 6.5 we can suppose that $D=D\left(\left\{n_{1}, n_{2}, \ldots, n_{t}\right\}\right)$ for some set $\left\{n_{1}, n_{2}, \ldots, n_{t}\right\}$ of elements in $D$. For each $n_{i}$ choose $z_{i}$ in $Z^{n_{i}}(E(\Lambda))$ such that $z_{i}$ is non-nilpotent.

Suppose now that $z$ is a non-nilpotent element in $Z^{n}(E(\Lambda))$ associated to $\mathcal{E}$. By choice of $\left\{n_{1}, n_{2}, \ldots, n_{t}\right\}$ there exist non-negative integers $\left\{c_{1}, c_{2}, \ldots, c_{t}\right\}$ such that $\sum_{i=1}^{t} c_{i} n_{i}=n$. Furthermore $z^{\prime}=z_{1}^{c_{1}} z_{2}^{c_{2}} \cdots z_{t}^{c_{t}}$ is a non-nilpotent element in $Z^{n}(E(\Lambda))$ associated to $\mathcal{E}$ by Lemma 6.4 . So from Proposition 6.3 , we have that $z=\alpha z^{\prime}$ in $Z_{\mathrm{gr}}(E(\Lambda)) / \mathcal{N}_{Z}$ for some $\alpha$ in $K \backslash\{0\}$. Thus $Z_{\mathrm{gr}}(E(\Lambda)) / \mathcal{N}_{Z}$ is a finitely generated $K$-algebra.

We have already observed that $Z_{\text {gr }}(E(\Lambda)) / \mathcal{N}_{Z}$ is a commutative $K$-algebra. Moreover, by Proposition 6.3(b) we have $\operatorname{dim}_{K} Z^{n}(E(\Lambda)) / \mathcal{N}_{Z}^{n} \leq r$ for some $r$ and for all $n \geq 0$. As $Z_{\mathrm{gr}}(E(\Lambda)) / \mathcal{N}_{Z}$ is a graded ring, the Krull dimension is equal to the rate of polynomial growth of $Z^{n}(E(\Lambda)) / \mathcal{N}_{Z}^{n}$. Hence the Krull dimension of $Z_{\text {gr }}(E(\Lambda)) / \mathcal{N}_{Z}$ is at most one.

We now give an example to show that $Z_{\mathrm{gr}}(E(\Lambda))$ need not be finitely generated, that is, the nilpotent part may be infinitely generated.

Example 6.7. Let $\Lambda=K \mathcal{Q} / I$ where $\mathcal{Q}$ is the quiver

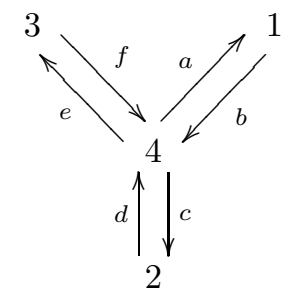

and

$$
I=\langle a b, b a, b c, c d, e f, f a\rangle .
$$

By direct calculations, one shows that $Z_{\mathrm{gr}}(E(\Lambda))$ is spanned by the identity and by the elements $g^{2 n+4}$ corresponding to the paths $R^{2 n+4}=e f(a b)^{n} c d$ for $n \geq 1$ as a vector space over $K$. Since $g^{2 n_{1}+4} g^{2 n_{2}+4}=0$ for all pairs of positive integers $n_{1}$ and $n_{2}$, the $K$-algebra $Z_{\mathrm{gr}}(E(\Lambda))$ is infinitely generated.

\section{The graded Centre And Hochschild COHOMOlOgy}

Let $\Lambda$ be an indecomposable finite dimensional $K$-algebra. Following [13], let $\mathcal{N}$ be the ideal in $\operatorname{HH}^{*}(\Lambda)$ generated by the homogeneous nilpotent elements. The Hochschild cohomology ring $\operatorname{HH}^{*}(\Lambda)$ of $\Lambda$ is graded commutative, so that $\mathrm{HH}^{*}(\Lambda) / \mathcal{N}$ is a commutative ring, in the same way as $Z_{\mathrm{gr}}(E(\Lambda)) / \mathcal{N}_{Z}$. As mentioned in the introduction, it was conjectured in [13] that $\mathrm{HH}^{*}(\Lambda) / \mathcal{N}$ is always a finitely generated $K$-algebra. In this section we show that this is indeed true whenever $\Lambda$ is a finite dimensional monomial algebra. Furthermore, in this case $\mathrm{HH}^{*}(\Lambda) / \mathcal{N}$ has Krull dimension at most one, and $\mathrm{HH}^{2 n}(\Lambda) /\left(\mathcal{N} \cap \mathrm{HH}^{2 n}(\Lambda)\right)$ is isomorphic to $Z^{2 n}(E(\Lambda)) /\left(\mathcal{N}_{Z} \cap Z^{2 n}(E(\Lambda))\right)$ for $n$ sufficiently large. Note that $\mathcal{N}_{Z} \cap Z^{2 n}(E(\Lambda))=\mathcal{N}_{Z}^{2 n}$. We end the section with a discussion on the relationship between $\mathrm{HH}^{*}(\Lambda)$ and $Z_{\mathrm{gr}}(E(\Lambda))$ in general and give an example showing that the ring homomorphism $\overline{\varphi_{\Lambda / \mathfrak{r}}}: \mathrm{HH}^{*}(\Lambda) / \mathcal{N} \rightarrow Z_{\mathrm{gr}}(E(\Lambda)) / \mathcal{N}_{Z}$ is not always onto.

Let $\Lambda=K \mathcal{Q} / I$ be an indecomposable finite dimensional monomial algebra. From Theorem 6.6 we have that $Z_{\mathrm{gr}}(E(\Lambda)) / \mathcal{N}_{Z}$ is a finitely generated $K$-algebra 
of Krull dimension at most one. Moreover, using Proposition 2.9, there is some $u \leq \operatorname{rl}(\Lambda)-1$ such that for each generator $z+\mathcal{N}_{Z}$, the element $z$ stabilizes at $u$ and $z^{u}+\mathcal{N}_{Z}$ is nonzero. We show first that each such $z^{u}+\mathcal{N}_{Z}$ (or $z^{u+1}+\mathcal{N}_{Z}$ if $u$ is odd) is in the image of the inclusion $\overline{\varphi_{\Lambda / \mathrm{r}}}: \mathrm{HH}^{*}(\Lambda) / \mathcal{N} \rightarrow Z_{\mathrm{gr}}(E(\Lambda)) / \mathcal{N}_{Z}$.

From [6] and Definition 1.13, recall that an element $R_{i}^{2 n+1}$ in $\mathcal{R}^{2 n+1}$ may be written uniquely as $R_{k}^{2 n} p$ and uniquely as $q R_{j}^{2 n}$ for some integers $j$ and $k$ and paths $p$ and $q$ in $K \mathcal{Q}$. We consider $\Lambda$ as a right $\Lambda^{e}$-module, and write $\otimes$ for $\otimes_{K}$. From [1], the map $\delta^{2 n+1}: P^{2 n+1} \rightarrow P^{2 n}$ in a minimal projective $\Lambda^{e}$-resolution $\left(P^{*}, \delta^{*}\right)$ of $\Lambda$, is given by

$$
\mathfrak{o}\left(R_{i}^{2 n+1}\right) \otimes \mathfrak{t}\left(R_{i}^{2 n+1}\right) \mapsto \mathfrak{o}\left(R_{k}^{2 n}\right) \otimes p-q \otimes \mathfrak{t}\left(R_{j}^{2 n}\right),
$$

where the first tensor lies in the summand $\Lambda \mathfrak{o}\left(R_{k}^{2 n}\right) \otimes \mathfrak{t}\left(R_{k}^{2 n}\right) \Lambda$ of $P^{2 n}$ and the second tensor lies in the summand $\Lambda \mathfrak{o}\left(R_{j}^{2 n}\right) \otimes \mathfrak{t}\left(R_{j}^{2 n}\right) \Lambda$ of $P^{2 n}$. If not specified, then it will always be clear from the context in which summand of a projective module our tensors lie.

Proposition 7.1. Keep the notation from above. Furthermore, let $\Lambda=K \mathcal{Q} / I$ be an indecomposable monomial algebra, and let $m$ be the even integer equal to $u$ or $u+1$. For each generator $z+\mathcal{N}_{Z}$ of $Z_{\mathrm{gr}}(E(\Lambda)) / \mathcal{N}_{Z}$, the element $z^{m}$ is in the image of $\phi_{\Lambda / \mathfrak{r}}$.

Proof. Let $m$ be the even integer equal to $u$ or $u+1$. Let $z+\mathcal{N}_{Z}$ be a non-zero homogeneous element of $Z_{\mathrm{gr}}(E(\Lambda)) / \mathcal{N}_{Z}$ associated to the equivalence class $\mathcal{E}$ and suppose that $z^{m}$ is in degree $2 n$. We may suppose that $z^{m}=\sum_{i \in \mathcal{I}} g_{i}^{2 n}$ where the $g_{i}^{2 n}$ are not nilpotent, all lie in the same equivalence class, and all $R_{i}^{2 n}$ are left and right $(2 n-1)$-stable. Moreover the $R_{i}^{2 n}$ are tightly packed and satisfy the properties of Proposition 4.7.

Let $\left(P^{*}, \delta^{*}\right)$ be a minimal projective resolution of $\Lambda$ as a right $\Lambda^{e}$-module. Then the summands of $P^{2 n}$ are indexed by the set $\mathcal{R}^{2 n}$. For $R^{2 n} \in \mathcal{R}^{2 n}$, define $\chi: P^{2 n} \rightarrow$ $\Lambda$ by

$$
\chi\left(\mathfrak{o}\left(R^{2 n}\right) \otimes \mathfrak{t}\left(R^{2 n}\right)\right)= \begin{cases}\mathfrak{o}\left(R_{i}^{2 n}\right) & \text { if } R^{2 n}=R_{i}^{2 n} \text { with } i \in \mathcal{I} \\ 0 & \text { otherwise. }\end{cases}
$$

It is clear that if $\chi$ represents an element in $\operatorname{HH}^{*}(\Lambda)$, then $\varphi_{\Lambda / \mathfrak{r}}(\chi)=z^{m}$. So, it is enough to show that $\chi$ represents an element of $\mathrm{HH}^{2 n}(\Lambda)$.

Fix some $R^{2 n+1} \in \mathcal{R}^{2 n+1}$. Consider the composite map

$$
\chi \delta^{2 n+1}: P^{2 n+1} \rightarrow P^{2 n} \rightarrow \Lambda
$$

and let $\Lambda \mathfrak{o}\left(R^{2 n+1}\right) \otimes \mathfrak{t}\left(R^{2 n+1}\right) \Lambda$ be an arbitrary summand of $P^{2 n+1}$. Write $R^{2 n+1}=$ $R_{i_{1}}^{2 n} p=q R_{i_{2}}^{2 n}$ for some paths $p$ and $q$.

If neither $i_{1}$ nor $i_{2}$ are in $\mathcal{I}$ then the restriction of $\chi \delta^{2 n+1}$ to the summand $\Lambda \mathfrak{o}\left(R^{2 n+1}\right) \otimes \mathfrak{t}\left(R^{2 n+1}\right) \Lambda$ is zero.

Now suppose that $i_{1} \in \mathcal{I}$, and without loss of generality suppose that $i_{1}=1$. Then, from Proposition 4.7, we may write $R_{1}^{2 n}=a_{1} r_{2,4} r_{2,6} \cdots r_{2,2 n} t_{1}$ where $t_{1} a_{1}=$ $r_{2,2}$.

From Proposition 5.5 we know that $R^{2 n+1}$ must lie on any walk in the equivalence class $\mathcal{E}$. Now $R_{1}^{2 n}=R_{1}^{2 n-1} t_{1}$ and there is a relation on the walk which has prefix $t_{1}$, namely $r_{2,2}$. So we have that $R^{2 n+1}=R_{1}^{2 n-1} t_{1} a_{1}=R_{1}^{2 n} a_{1}$ and hence $p=a_{1}$. 
Then

$$
\begin{aligned}
R_{1}^{2 n} a_{1} & =\left(a_{1} r_{2,4} r_{2,6} \cdots r_{2,2 n} t_{1}\right) a_{1} \\
& =a_{1}\left(r_{2,4} r_{2,6} \cdots r_{2,2 n} r_{2,2}\right) \\
& =a_{1} R_{j}^{2 n}
\end{aligned}
$$

for some $j \in \mathcal{I}$ by Lemma 4.3. Hence $q=a_{1}$ and the map $\delta^{2 n+1}$ is given on this summand by

$$
\delta^{2 n+1}\left(\mathfrak{o}\left(R^{2 n+1}\right) \otimes \mathfrak{t}\left(R^{2 n+1}\right)\right)=\mathfrak{o}\left(R_{1}^{2 n}\right) \otimes a_{1}-a_{1} \otimes \mathfrak{t}\left(R_{j}^{2 n}\right) .
$$

Hence

$$
\chi \delta^{2 n+1}\left(\mathfrak{o}\left(R^{2 n+1}\right) \otimes \mathfrak{t}\left(R^{2 n+1}\right)\right)=a_{1}-a_{1}=0 .
$$

Thus $\chi \in \mathrm{HH}^{*}(\Lambda)$.

The final case where $i_{2} \in \mathcal{I}$ is similar. This completes the proof.

This has the following immediate consequence.

Proposition 7.2. Let $\Lambda=K \mathcal{Q} / I$ be an indecomposable monomial algebra. Then the ring homomorphism $\varphi_{\Lambda / \mathfrak{r}}$ induces an isomorphism

$$
\mathrm{HH}^{2 n}(\Lambda) /\left(\mathcal{N} \cap \mathrm{HH}^{2 n}(\Lambda)\right) \cong Z^{2 n}(E(\Lambda)) /\left(\mathcal{N}_{Z} \cap Z^{2 n}(E(\Lambda))\right)
$$

for some positive integer $N$ and for all $n \geq N$.

In particular, when the characteristic of $K$ is different from two, this shows that the map $\overline{\varphi_{\Lambda / \mathrm{r}}}: \mathrm{HH}^{*}(\Lambda) / \mathcal{N} \rightarrow Z_{\mathrm{gr}}(E(\Lambda)) / \mathcal{N}_{Z}$ is an isomorphism beyond a certain degree. If $z$ is a non-nilpotent element in $Z_{\mathrm{gr}}(E(\Lambda))$ of odd degree, then $z^{2 i} z$ is in the $\mathrm{HH}^{*}(\Lambda)$-submodule of $Z_{\mathrm{gr}}(E(\Lambda)) / \mathcal{N}_{Z}$ generated by $z$ for sufficiently large $i$. Using this it follows that $Z_{\mathrm{gr}}(E(\Lambda)) / \mathcal{N}_{Z}$ is a finitely generated $\mathrm{HH}^{*}(\Lambda)$-module.

Combining the results that we have obtained so far, we prove the main result of the paper.

Theorem 7.3. Let $\Lambda=K \mathcal{Q} / I$ be an indecomposable monomial algebra. Then $\mathrm{HH}^{*}(\Lambda) / \mathcal{N}$ is a commutative finitely generated $K$-algebra of Krull dimension at most one.

Proof. The map $\overline{\varphi_{\Lambda / \mathrm{r}}}: \mathrm{HH}^{*}(\Lambda) / \mathcal{N} \rightarrow Z_{\mathrm{gr}}(E(\Lambda)) / \mathcal{N}_{Z}$ is an inclusion, so that

$$
\operatorname{dim}_{K} \mathrm{HH}^{n}(\Lambda) /\left(\mathcal{N} \cap \mathrm{HH}^{n}(\Lambda)\right) \leq \operatorname{dim}_{K} Z^{n}(E(\Lambda)) /\left(\mathcal{N}_{Z} \cap Z^{n}(E(\Lambda))\right) \leq N
$$

for some integer $N$ and for all $n \geq 0$. As $\operatorname{HH}^{*}(\Lambda) / \mathcal{N}$ is a commutative graded ring, it follows that the Krull dimension is at most one.

If the Krull dimension is zero, there is nothing left to prove. So suppose that the Krull dimension of $\operatorname{HH}^{*}(\Lambda) / \mathcal{N}$ is one. The Krull dimension of $Z_{\mathrm{gr}}(E(\Lambda)) / \mathcal{N}_{Z}$ is also one and this is a finitely generated $K$-algebra, so that by the Noether Normalisation Theorem there exists a homogeneous element $\bar{z}=z+\mathcal{N}_{Z}$ in $Z_{\mathrm{gr}}(E(\Lambda)) / \mathcal{N}_{Z}$ such that $Z_{\mathrm{gr}}(E(\Lambda)) / \mathcal{N}_{Z}$ is a finitely generated module over the subring $K[\bar{z}]$. Using Proposition 7.1 there exists an element $\eta$ in $\mathrm{HH}^{*}(\Lambda)$ such that $\overline{\varphi_{\Lambda / \mathfrak{r}}}(\eta)=\bar{z}^{m}$ for some positive integer $m$. Then it easy to see that $Z_{\mathrm{gr}}(E(\Lambda)) / \mathcal{N}_{Z}$ is a finitely generated module over the subring $K[\bar{\eta}]$ of $\mathrm{HH}^{*}(\Lambda) / \mathcal{N}$. Since $\mathrm{HH}^{*}(\Lambda) / \mathcal{N}$ is a $K[\bar{\eta}]-$ submodule of $Z_{\mathrm{gr}}(E(\Lambda)) / \mathcal{N}_{Z}$, the module $\mathrm{HH}^{*}(\Lambda) / \mathcal{N}$ is a finitely generated $K[\bar{\eta}]-$ module. It is now immediate that $\operatorname{HH}^{*}(\Lambda) / \mathcal{N}$ is a finitely generated $K$-algebra. 
Thus the conjecture made in [13] holds for all indecomposable finite dimensional monomial algebras $\Lambda=K \mathcal{Q} / I$. In [8] even stronger results are obtained for a class of algebras that generalises Koszul monomial algebras, namely for $(D, A)$-stacked monomial algebras. In that case the precise description and number of generators of $\operatorname{HH}^{*}(\Lambda) / \mathcal{N}$ is also found.

Now let $\Lambda$ be any finite dimensional $K$-algebra. The proof of the main result of this paper heavily depended on our special knowledge of $\operatorname{Im} \varphi_{\Lambda / \mathfrak{r}}$ as a subring of $E(\Lambda)$. The precise relationship between $\operatorname{Im} \varphi_{\Lambda / \mathfrak{r}}$ and $E(\Lambda)$ has been found by Bernhard Keller [12, Theorem 3.5]. The Ext algebra of any module is endowed with an $\mathbb{A}_{\infty}$-structure (see [11]), so using this $\mathbb{A}_{\infty}$-structure for $E(\Lambda)$, one defines a centre $Z_{\infty}(E(\Lambda))$ of $E(\Lambda)$ as an $\mathbb{A}_{\infty}$-algebra. Keller has shown that $\operatorname{Im} \varphi_{\Lambda / \mathfrak{r}}=Z_{\infty}(E(\Lambda))$. Hence our results show that, modulo nilpotence, $Z_{\mathrm{gr}}^{2 n}(E(\Lambda))$ and $Z_{\infty}^{2 n}(E(\Lambda))$ are isomorphic for all $n \geq N$ for some $N$ whenever $\Lambda$ is a finite dimensional monomial algebra.

The Ext algebra $E(\Lambda)$ has a trivial $\mathbb{A}_{\infty}$-structure when $\Lambda$ is a Koszul algebra, so that in this case $Z_{\infty}(E(\Lambda))=Z_{\mathrm{gr}}(E(\Lambda))$ and $\varphi_{\Lambda / \mathrm{r}}: \mathrm{HH}^{*}(\Lambda) \rightarrow Z_{\mathrm{gr}}(E(\Lambda))$ is surjective. This was first independently observed by Green-Snashall-Solberg for Koszul path algebras and for more general Koszul algebras by Ragnar-Olaf Buchweitz [2], and then subsequently generalised by Keller as referred to above [12].

The algebra $\Lambda=K \mathcal{Q} / J^{2}$ is a Koszul algebra for any finite quiver $\mathcal{Q}$ with $J$ denoting the ideal generated by all the arrows in $\mathcal{Q}$. Here $E(\Lambda) \cong K \mathcal{Q}$. These algebras were considered in [3]. Using the following lemma we use the above observations to obtain some of the structural information on the Hochschild cohomology ring of $K \mathcal{Q} / J^{2}$ given there.

Lemma 7.4. Let $\Lambda$ be a finite dimensional $K$-algebra such that $\Lambda / \mathfrak{r} \otimes_{K} \Lambda / \mathfrak{r}$ is semisimple. Then $\left(\operatorname{Ker} \varphi_{\Lambda / \mathrm{r}}\right)^{\mathrm{r}(\Lambda)}=(0)$.

Proof. Let $\eta$ be a homogeneous element in $\operatorname{Ker} \varphi_{\Lambda / \mathfrak{r}}$ of degree $m$. In the proof of Proposition 4.4 in [13] it is shown that $\operatorname{Im} \Omega_{\Lambda^{e}}^{n}(\eta) \subseteq \mathfrak{r} \Omega_{\Lambda^{e}}^{n}(\Lambda)+\Omega_{\Lambda^{e}}^{n}(\Lambda) \mathfrak{r}$ for any $n \geq$ 0 . So if $\eta^{\prime}$ is a homogeneous element in $\operatorname{Ker} \varphi_{\Lambda / \mathfrak{r}}$ of degree $n$, then $\operatorname{Im}\left(\eta \Omega_{\Lambda^{e}}^{m}\left(\eta^{\prime}\right)\right) \subseteq$ $\mathfrak{r}^{2}$. Equivalently, $\operatorname{Im}\left(\eta \cdot \eta^{\prime}\right) \subseteq \mathfrak{r}^{2}$. By induction we have that $\operatorname{Im}\left(\eta_{1} \cdot \eta_{2} \cdots \eta_{t}\right) \subseteq \mathfrak{r}^{t}$ for any set of homogeneous elements $\left\{\eta_{1}, \eta_{2}, \ldots, \eta_{t}\right\}$ in $\operatorname{Ker} \varphi_{\Lambda / \mathfrak{r}}$. Elements of degree zero in $\operatorname{Ker} \varphi_{\Lambda / \mathfrak{r}}$ are in the radical of the centre of $\Lambda$, which is contained in $\mathfrak{r}$. The claim follows from these observations.

It is easy to see that $Z_{\mathrm{gr}}(K \mathcal{Q}) \cong K$ whenever $\mathcal{Q}$ is not an oriented cycle (not equal to $\widetilde{\mathbb{A}}_{n}$ with cyclic orientation for any $n$ ). Again let $\Lambda=K \mathcal{Q} / J^{2}$. Hence if $\mathcal{Q}$ is not an oriented cycle, then $\operatorname{Ker} \varphi_{\Lambda / \mathfrak{r}}=\operatorname{HH}^{\geq 1}(\Lambda) \cup \operatorname{radHH}^{0}(\Lambda)$. Using the above lemma we obtain that any product of two elements in $\operatorname{HH}^{\geq 1}(\Lambda) \cup \operatorname{rad} \operatorname{HH}^{0}(\Lambda)$ is zero. For further results see [3].

We end this paper with two examples. The first example shows that the map $\bar{\varphi}_{\Lambda / \mathfrak{r}}: \mathrm{HH}^{*}(\Lambda) / \mathcal{N} \rightarrow Z_{\mathrm{gr}}(E(\Lambda)) / \mathcal{N}_{Z}$ can be an isomorphism even though the stability condition in Proposition 7.1 is not satisfied. The last example provides a case where the map $\bar{\varphi}_{\Lambda / \mathfrak{r}}$ is not onto, or equivalently, is not an isomorphism.

Example 7.5. Consider the Example 4.1 again. The element $z=g_{1}^{4}+g_{2}^{4}+g_{3}^{4}+g_{4}^{4}$ is non-nilpotent in $Z_{\mathrm{gr}}(E(\Lambda))$ and $Z_{\mathrm{gr}}(E(\Lambda)) / \mathcal{N}_{Z} \cong K[\bar{z}]$. Moreover $z$ has degree 4 , and it is left and right 7 -stable. So the point $u$ where $z$ stabilizes is 2 , and therefore $\bar{z}^{2}$ is in the image of $\bar{\varphi}_{\Lambda / \mathfrak{r}}$ by Proposition 7.1. However we claim that $\bar{z}$ is in the 
image of $\bar{\varphi}_{\Lambda / \mathfrak{r}}$. Let $P^{*}: \cdots \rightarrow P^{n} \rightarrow P^{n-1} \rightarrow \cdots \rightarrow P^{1} \rightarrow P^{0} \rightarrow \Lambda \rightarrow 0$ be a minimal projective $\Lambda^{e}$-projective resolution of $\Lambda$. Define $\chi: P^{4} \rightarrow \Lambda$ by

$$
\chi\left(\mathfrak{o}\left(R_{j}^{4}\right) \otimes \mathfrak{t}\left(R_{j}^{4}\right)\right)= \begin{cases}\mathfrak{o}\left(R_{j}^{4}\right) & \text { for } j=1,2,3,4 ; \\ a_{2} & \text { for } j=5 .\end{cases}
$$

Then $\chi$ is in $\operatorname{HH}^{4}(\Lambda)$ and $\varphi_{\Lambda / \mathfrak{r}}(\chi)=z$, so that $\overline{\varphi_{\Lambda / \mathfrak{r}}}: \mathrm{HH}^{*}(\Lambda) / \mathcal{N} \rightarrow Z_{\mathrm{gr}}(E(\Lambda)) / \mathcal{N}_{Z}$ is an isomorphism.

Example 7.6. Let $\Lambda=K \mathcal{Q} / I$ where $\mathcal{Q}$ is the quiver

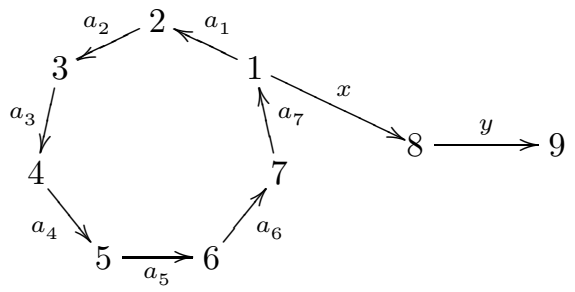

and

$$
I=\left\langle a_{1} a_{2} a_{3} a_{4}, a_{2} a_{3} a_{4} a_{5}, a_{4} a_{5} a_{6}, a_{5} a_{6} a_{7}, a_{6} a_{7} a_{1} a_{2}, a_{6} a_{7} x y, a_{7} a_{1} a_{2} a_{3}\right\rangle .
$$

It is easy to see that $R_{1}^{4}=a_{1} a_{2} a_{3} a_{4} a_{5} a_{6} a_{7}, R_{2}^{4}=a_{4} a_{5} a_{6} a_{7} a_{1} a_{2} a_{3}, R_{3}^{4}=$ $a_{5} a_{6} a_{7} a_{1} a_{2} a_{3} a_{4}$, and $R_{4}^{4}=a_{7} a_{1} a_{2} a_{3} a_{4} a_{5} a_{6}$ are all in $\mathcal{R}^{4}$. Moreover $z=g_{1}^{4}+$ $g_{2}^{4}+g_{3}^{4}+g_{4}^{4}$ is in $Z_{\mathrm{gr}}(E(\Lambda))$ in degree 4 . Since one can check that $\operatorname{HH}^{4}(\Lambda)=(0)$, the induced map $\overline{\varphi_{\Lambda / \mathrm{r}}}: \mathrm{HH}^{*}(\Lambda) / \mathcal{N} \rightarrow Z_{\mathrm{gr}}(E(\Lambda)) / \mathcal{N}_{Z}$ is not onto. To this end note that $g_{1}^{4}$ is not stable.

\section{REFERENCES}

[1] Bardzell, M. J., The alternating syzygy behavior of monomial algebras, J. Algebra 188 (1997), 69-89.

[2] Buchweitz, R.-O., Hochschild cohomology of Koszul algebras, Talk at the Conference on Representation Theory, Canberra, July 2003.

[3] Cibils, C., Hochschild cohomology algebra of radical square zero algebras, Algebras and modules, II (Geiranger, 1996), 93-101, CMS Conf. Proc., 24, Amer. Math. Soc., Providence, RI, 1998.

[4] Evens, L., The cohomology ring of a finite group, Trans. Amer. Math. Soc. 101 (1961), 224-239.

[5] Friedlander, E. M. and Suslin, A., Cohomology of finite group schemes over a field, Invent. Math. 127 (1997), no. 2, 209-270.

[6] Green, E. L., Happel, D. and Zacharia, D., Projective resolutions over Artin algebras with zero relations, Illinois J. Math. 29 (1985), no. 1, 180-190.

[7] Golod, E., The cohomology ring of a finite p-group, Dokl. Akad. Nauk SSSR, 125 (1959) 703-706.

[8] Green, E. L. and Snashall, N., The Hochschild cohomology ring modulo nilpotence of a stacked monomial algebra, Preprint 2004.

[9] Green, E. L., Snashall, N. and Solberg, Ø., The Hochschild cohomology ring of a selfinjective algebra of finite representation type, Proc. Amer. Math. Soc. 131 (2003), no. 11, 3387-3393.

[10] Green, E. L. and Zacharia, D., The cohomology ring of a monomial algebra, Manuscripta Math. 85 (1994), no. 1, 11-23.

[11] Keller, B., A-infinity algebras in representation theory, Proceedings of ICRA IX, Beijing 2000.

[12] - Derived invariance of higher structures on the Hochschild complex, Preprint 2003.

[13] Snashall, N. and Solberg, Ø., Support varieties and Hochschild cohomology rings, to appear in Proc. London Math. Soc. 
[14] Venkov, B. B., Cohomology algebras for some classifying spaces, Dokl. Akad. Nauk SSSR 127 (1959), 943-944.

Edward L. Green, Department of Mathematics, Virginia Tech, Blacksburg, VA 240610123, USA

E-mail address: green@math.vt.edu

Nicole Snashall, Department of Mathematics, University of Leicester, University RoAd, Leicester, LE1 7RH, England

E-mail address: N.Snashall@mcs.le.ac.uk

Øyvind Solberg, Institutt for matematiske fag, NTNU, N-7491 Trondheim, Norway

E-mail address: oyvinso@math.ntnu.no 